\title{
A $\delta$-plane simulation of anticyclones perturbing circumpolar flows to form a transient north polar hexagon
}

\author{
J. M. Cosgrove` and L. K. Forbes \\ School of Mathematics and Physics, University of Tasmania, PO Box 37, Hobart, 7001 Tasmania, Australia
}

Accepted 2017 May 10. Received 2017 May 10; in original form 2017 February 1

\begin{abstract}
Saturn's North Polar Hexagon was discovered by Godfrey who pieced together map projections of images captured by the Voyager mission to unveil a hexagonal structure over the north pole of Saturn. This article attempts to answer whether or not a hexagonal structure can be formed through anticyclones impinging on the dominant eastward circumpolar flow and is in part based upon the proposed theory by Allison et al. that the Hexagon may be the result of at least one impinging anticyclone perturbing a circumpolar jet centrally located around the $76^{\circ} \mathrm{N}$ latitude. A high-latitude $\delta$-plane approximation is used to simulate the interaction between an initially circular circumpolar jet and at least one perturbing anticyclone. Our simulations with one perturbing anticyclone failed to form a hexagonal structure; yet by including an additional anticyclone it was found that depending on the strength, location and radius of the perturbing anticyclones a hexagonal feature could develop. However, the longevity and drift rate of the actual Hexagon must be attributed to other factors not considered in this paper.
\end{abstract}

Key words: hydrodynamics - planets and satellites: atmospheres.

\section{INTRODUCTION}

The Voyager space probes that were launched in 1977 heralded vast scientific insight into the study of the outer Solar system. Originally the objective was to obtain data on the atmospheric conditions of Jupiter and Saturn, their satellites and the rings of Saturn (Smith et al. 1977). It was envisaged that the Voyager flight paths would enable observations of the north and south poles of Saturn's atmosphere. However, due to the south pole being in total darkness at the time, images of the south pole could not be captured (Godfrey 1988). The north polar images on the other hand revealed a hexagonal structure centred at the north pole. Godfrey (1988) pieced together map-projections taken as Saturn rotated and thus was the first to identify Saturn's North Polar Hexagon. Fig. 1 is the sixth and final mosaic published in Godfrey's paper. Godfrey (1988) also produced streamline maps that showed that there existed a hexagonal flow pattern about the pole, with a substantial counter-rotating vortex along one edge. It is also clear from these streamline plots that there are other counter-rotating vortices that were not visible, aligned along the other edges of the Hexagon.

The Hexagon appears almost stationary with respect to the internal rotation of Saturn (Godfrey 1990) and is ingrained in an eastward circumpolar jet of width $4^{\circ}$ latitude. However, the cloud formations within the hexagonal structure were observed by the Voyager space craft to move with a velocity of approximately $100 \mathrm{~m} \mathrm{~s}^{-1}$ at the mid-point latitude of $76^{\circ}$.

There has been some considerable work on interpreting the origin of the North Polar Hexagon on Saturn; Allison, Godfrey \& Beebe (1990) proposed that the Hexagon is the result of the eastward jet, in the form of a planetary Rossby wave being continually perturbed by at least one anticyclonic elliptical vortex to the south of the structure. This proposition is in part based on the fact that an anticyclone with approximate radius $3000 \mathrm{~km}$ is observed to be visibly impinging on one edge of the Hexagon in the mosaic published by Godfrey (1988) and thus has been of considerable interest and widely cited. Godfrey (1990) estimated that the visible vortex has a rotation rate of $-8.13 \pm 0.6 \times 10^{-9} \mathrm{rad} \mathrm{s}^{-1}$ with respect to a fixed meridian, in later publications the rotation rate of the once visible vortex is referred to as the drift rate. The drift rate calculated by Godfrey (1990) corresponds to roughly $-0.040^{\circ} \mathrm{d}^{-1}$ using data captured over a nine-month period during 1980 and 1981.

Until the early 1990s, it was not established whether the rotation rate estimated by Godfrey (1990) was in fact representative of the actual movement of the vortex, or just error that could be associated with the internal rotation rate of Saturn (Caldwell et al. 1993). The Hubble Space Telescope (HST) observations in 1990 and 1991 confirmed that there is movement of the visible vortex relative to the rotation of Saturn. Caldwell et al. (1993) coupled the data interpreted from the original Voyager images and the HST observations to form an 11-yr baseline. It was found that over this longer period the vortex moved $-0.0569^{\circ} \mathrm{d}^{-1}$. 


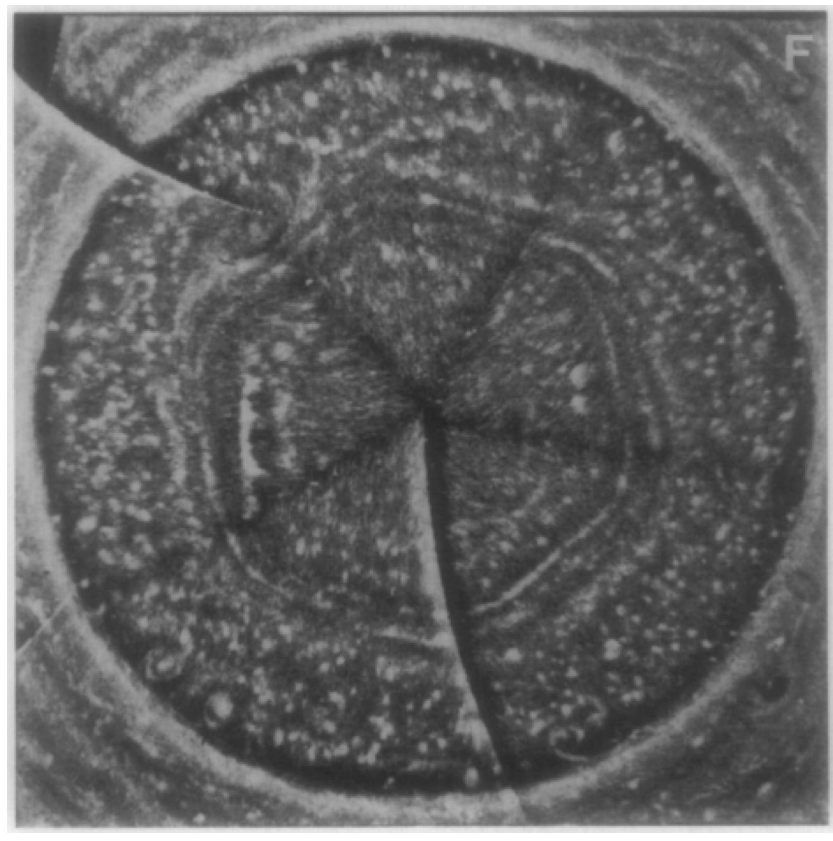

Figure 1. Composite map-projected images of Saturn's North Polar Hexagon as published by Godfrey (1988) and reprinted with permission from Elsevier.

The Hexagon and visible vortex were also observed over the period 1990 July to 1991 October using ground-based instruments (Sanchez-Lavega et al. 1993). Over this 16-month period the vortex drifted longitudinally at a mean rate of $-0.0353^{\circ} \mathrm{d}^{-1}$. These data were collated with the observational data used by Godfrey (1990) to give another perspective into the long-term motion of the vortex. The long-term drift rate in this case was calculated to be $-0.0577^{\circ} \mathrm{d}^{-1}$ (Sanchez-Lavega et al. 1993), nearly identical to that of the HST value calculated by Caldwell et al. (1993). Although the average drift rates of the visible vortex given by Godfrey (1990), Caldwell et al. (1993) and Sanchez-Lavega et al. (1993) are relatively small with respect to Saturn's internal rotation, the short-term fluctuation is considerable, drifting upwards of $14^{\circ}$ longitudinally at rates of approximately $1^{\circ} \mathrm{d}^{-1}$, as well as latitudinal movement but on a much smaller scale (Sanchez-Lavega et al. 1993).

Interestingly, Sanchez-Lavega et al. (1993) raise the possibility that the vortex observed using ground-based technology in 1990 may be a different vortex than that captured by the Voyager spacecraft. This conclusion is based on knowing the longitude of the vortex at the time when the Voyager and 1990 images were captured and projecting forward in time using the respective short-term drift rates of $-0.040^{\circ}$ (Godfrey 1988) and $-0.0353^{\circ}$ (SanchezLavega et al. 1993) $\mathrm{d}^{-1}$. It was shown that the vortex would be in two distinct locations separated by approximately $60^{\circ}$ in longitude, the angle spanned by a hexagon side. This therefore led SanchezLavega et al. (1993) to suggest that there might be at least two vortices centrally located along the edge of the Hexagon that may or may not be visible at different times. This theory would coincide with the streamline maps produced by Godfrey (1988) and that of Allison et al. (1990) who proposed possible multiple perturbing anticyclones.

Images captured by the visual-infrared mapping spectrometer (VIMS) on board the Cassini-Huygens Orbiter in 2006 (Baines et al. 2009), and more recently the first visible light images captured by the Imaging Science Subsystem in 2009 (Sayanagi,
Ewald \& Ingersoll 2009) indicate that the Hexagon is a long-lived atmospheric vortex. However, these images indicate that the previously observed anticyclone(s) in 1980 and 1990 have dissipated to non-existence, casting doubt over the Allison et al. (1990) proposition. Although there is currently no visible anticyclonic vortex to the south of the Hexagon, there is a visible anticyclonic spot located in the interior of the Hexagon (Antuñano et al. 2015; Morales-Juberias et al. 2015), indicating that anticyclonic phenomena occur on a regular basis to the north and south of the Hexagon. The hexagonal structure bounds a pole centred cyclonic vortex with speed upwards of $135 \mathrm{~m} \mathrm{~s}^{-1}$ (Baines et al. 2009; Antuñano et al. 2015).

The VIMS imagery indicates that the clouds within the easterly flow have speeds in excess of $125 \mathrm{~m} \mathrm{~s}^{-1}$, an increase of 25 per cent from the observations of Voyager. These observed changes could be due to temporal variability and/or vertical shear (Baines et al. 2009). The most recent observations suggest that the peak velocity within the Hexagon is around $119 \mathrm{~m} \mathrm{~s}^{-1}$ (Antuñano et al. 2015).

Rotating tank experiments have been used for simulating atmospheric jets that meander about a given latitude. An interesting and not uncommon result of such experiments is that various polygonal flow features form, depending on the type of forcing (Sommeria, Meyers \& Swinney 1989; Vatistas 1990; Vatistas, Wang \& Lin 1994; Marcus \& Lee 1998; Jansson et al. 2006). This type of experiment has given rise to alternate explanations of the origin of the North Polar Hexagon. In particular, laboratory experiments with fluids in rotating tanks in conjunction with a barotropic linear instability analysis of Saturn's zonal wind profile were used to infer that Saturn's Hexagon is the result of equilibrated wavemodes of the barotropic instability, with mode six being the preferred state on Saturn due to the location and strength of the eastward circumpolar jet (Barbosa-Aguiar et al. 2010). Numerical simulations using the Explicit Planetary Isentropic-Coordinate (EPIC) model found that a stable hexagonal structure can result without forcing (MoralesJuberias et al. 2011). The EPIC simulations are in agreement with the experimental findings of Barbosa-Aguiar et al. (2010) and the resulting hexagonal structure arises due to the formation of what is termed a 'vortex street', where there is an alignment of opposing vortices such that there exists a meandering jet separating them. In the case of the Saturn hexagon simulations, there are six anticyclones slightly south of the six cyclones.

Although the experimental (Barbosa-Aguiar et al. 2010) and numerical (Morales-Juberias et al. 2011) results produce hexagonal features as a bi-product of a 'vortex street', there remains doubt over the legitimacy of such a model producing the actual North Polar Hexagon on Saturn, due to the debate over the existence of large vortices being observed in the vicinity of the Hexagon region of Saturn (Morales-Juberias et al. 2011; Sanchez-Lavega et al. 2014). A visible 'vortex street' may not be apparent in the Cassini images (Sanchez-Lavega et al. 2014), but it has been shown previously by analysing the original Voyager images that there was a visible anticyclone and several other non-visible anticyclonic regions aligned centrally along the southern side of the Hexagon edges (Godfrey 1988), but the lack of cyclonic regions to the north exclude it from being the result of a 'vortex street'. The speed needed to propagate the 'vortex street' hexagon in the numerical simulations by Morales-Juberias et al. (2011) would exceed the statistical bounds of measurements by Godfrey (1988). However, by considering small perturbations to an eastward Gaussian jet similar to that used by Morales-Juberias et al. (2011), a hexagon can result in the form of a shallow meandering jet that has comparable phase speed to the actual North Polar Hexagon (Morales-Juberias et al. 2015). 
A common practice in simulating atmospheric phenomena is to simplify spherical coordinate geometry by projecting on to a 'tangent plane'. The $f$-plane and $\beta$-plane approximations are used extensively in atmospheric fluid dynamics, but their validation is restricted to mid-latitude regions (Lipps 1964; Vallis 2006). In simulating the North Polar Hexagon, a high-latitude polar approximation must be used. In order to study high-latitude atmospheric Rossby waves, Yang (1987) proposed a $\delta$-plane approximation. Similar to the development of the $\beta$-plane where the $\beta$ term represents the rate of change in the Coriolis parameter $f$, the $\delta$-plane (denoted $\gamma$-plane by Nof 1990) includes the latitudinal variation of $\beta$ so that $\delta=\partial \beta / \partial y$ and thus allows for the quadratic variation in the Coriolis parameter around the polar regions. The quadratic variability in the Coriolis parameter was noted by LeBlond (1964) in what is considered to be the first valid polar plane approximation technique.

Saturn's Hexagon has the north pole centrally located within and thus the pole itself is the logical tangent point for the $\delta$-plane approximation. Nof (1990) outlines the appropriate momentum equations to use in such an instance and has the quadratic term in the form $\left(x^{2}+y^{2}\right)$, indicating that lines of latitude are circles centred about the pole, with zero linear variation term; $\beta=0$. These equations are also extended to take into account that not all polar approximations have the need for the pole to be the tangent point and a simple coordinate transformation enables the $\beta$ term to be recovered so that the Coriolis parameter also has linear variation in latitude. A similar approach was used by Harlander, Schonfeldt \& Metz (2000) to investigate flows near the poles with a poleward rigid boundary, thereby only having linear and quadratic change along the north-south $y$-axis. Unlike the $\beta$-plane approximation, higher order approximations such as the $\delta$-plane theories outlined by Yang (1987), Nof (1990) and Harlander et al. (2000) cannot be derived from spherical geographic coordinates. However, using a rotated geographic coordinate system, Harlander (2005) was able to derive a $\delta$-plane model from spherical geometry.

The current non-existence of a perturbing anticyclone has for at least a decade now cast doubt over the original proposal of Allison et al. (1990). However, this does not necessarily mean that the former visible spots impinging on the hexagonal edges did not influence its development initially, conversely the visible spots may have been the result of the Hexagon itself. It cannot be denied that at the time of discovery, the Hexagon had a significant counter-rotating vortex pushing on one edge. Without data prior to 1980 it is hard to say how long the spot was in existence. Maybe these vortices were enough to give the circumpolar jet the required perturbation similar to those given to the model used by Morales-Juberias et al. (2015) so that the circumpolar current could evolve to form a hexagonal structure. Although not correct, the theory of Allison et al. (1990) and the multiple vortex conjecture discussed by Sanchez-Lavega et al. (1993) raises some interesting questions and thus this article seeks to determine whether or not a hexagonal feature can be formed by anticyclones perturbing an initially circular circumpolar jet. A $\delta$-plane approximation similar to the one outlined by Nof (1990) will be used to simulate the spheroidal effects of polar flow and a Gaussian profile function will represent both the predominant eastward circumpolar current and the smaller magnitude westward flow to the south.

\section{MATHEMATICAL FORMULATION}

A two-dimensional Cartesian coordinates system is used to simulate vortex phenomena over the north pole of Saturn. However, the $x$ - and $y$-axes are not defined to point east and north, respectively. The origin of the coordinate system is the north pole.

The motion of the atmospheric fluid is driven by the Coriolis pseudo-force. The Coriolis parameter at latitude $\phi$ is given by

$f=2 \Omega \sin \phi$,

where $\Omega$ is the angular velocity of Saturn. The sidereal rotation period of Saturn is taken to be $10.656 \mathrm{~h}$, corresponding to the Saturnian system III coordinates (Williams 2015) and thus Saturn's angular velocity is

$\Omega=\frac{2 \pi}{10.656 \times 3600}=1.638 \times 10^{-4} \mathrm{~s}^{-1}$.

The $\delta$-plane approximation assumes that the Coriolis parameter $f$ has quadratic variability, so that

$f=2 \Omega-\delta\left(x^{2}+y^{2}\right)$,

where $2 \Omega$ is the value of the Coriolis parameter given by equation (1) at the north pole latitude and $\delta$ is the absolute value of the second derivative of (1) with respect to the latitude $\phi$ evaluated at the north pole and is given by

$\delta=\frac{\Omega}{R_{\mathrm{s}}^{2}}$,

in which $R_{\mathrm{S}}$ is the radius of Saturn. The $\delta$-plane has the unique characteristic that the magnitude of Coriolis parameter decreases away from the pole, which forms the origin of this 'tangent plane'. Unlike the more commonly used $f$-plane and $\beta$-plane, the $x$ - and $y$-axes are not defined to be pointing east and north, respectively. The easterly direction is defined to be pointing anticlockwise around circles of equal latitude with centres located at the origin, and the northerly direction is the ray from any $(x, y)$ coordinate terminating at the pole. A geometric sketch of the dimensional $\delta$-plane is given in Fig. 2, with boundary $-L<x<L,-H<y<H$. The arrows along the rays terminating at the origin show the northerly direction and the arrows on the concentric circles show the easterly direction. It is important to note that the $\delta$-plane approximation does not describe dynamics on a hypothetical tangent plane and only takes the name from the analogous $f$-plane approximation.

It is convenient to scale the problem so that all quantities, variables and equations are dimensionless. Pressure is referenced to the quantity $P_{0}=\rho_{0} R T_{0}$, in which $R$ is the ideal gas constant and takes the values of $R=4016.43 \mathrm{~J} \mathrm{~kg}^{-1} \mathrm{~K}^{-1}$ on Saturn and $\rho_{0}$ and

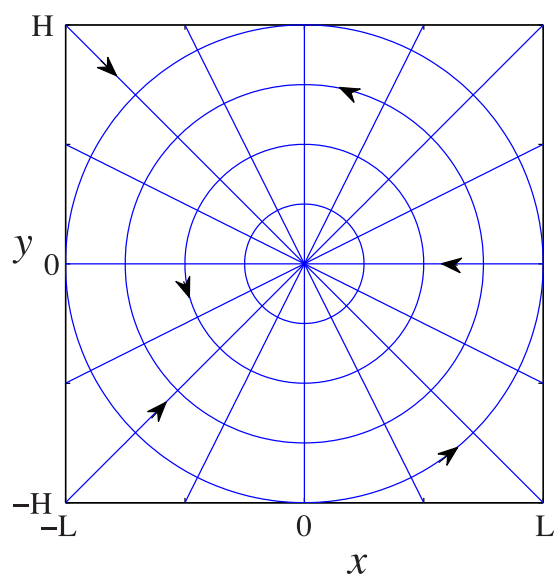

Figure 2. Geometric sketch of dimensional $\delta$-plane with north and east directional arrows. 


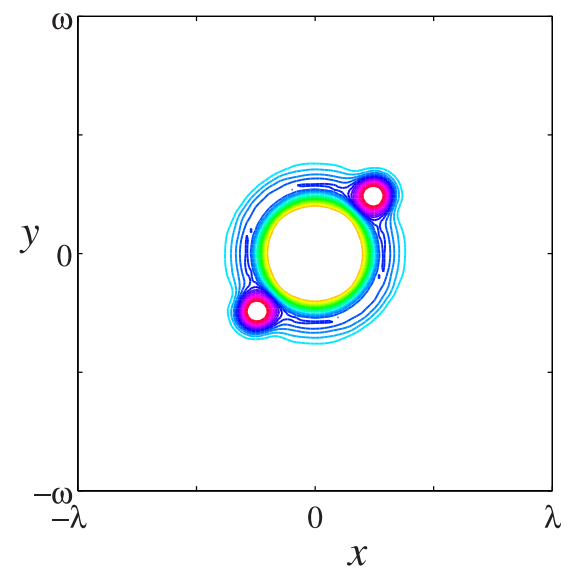

Figure 3. Sketch of the dimensionless computational $\delta$-plane. The contours have been taken from an actual solution of the pressure at time $t=0.1$.

$T_{0}$ are the respective scaling values for the density and temperature. The time-scale is $\tau$, the approximate number of seconds in one day $\left(10^{5} \mathrm{~s}\right)$. The length-scale $D$ is the approximate distance spanned by $10^{\circ}$ of latitude $\left(10^{7} \mathrm{~m}\right)$. It follows then that the speed is scaled relative to $D / \tau$, the approximate maximum velocity inside the hexagonal flow. The problem then is found to be dependent on six dimensionless constant parameters,

$\lambda=\frac{L}{D}, \quad \omega=\frac{H}{D}, \quad \gamma=\frac{c_{\mathrm{p}}}{c_{\mathrm{v}}}$,

$v=\frac{\tau^{2} R T_{0}}{D^{2}}, \quad \hat{\delta}=\delta A^{2} \tau, \quad \hat{\Omega}=\Omega \tau$.

The first two constants $\lambda$ and $\omega$ in the system (5) represent the dimensionless half-width and half-breadth of the tangent plane, respectively. The constant $\gamma=c_{\mathrm{p}} / c_{\mathrm{v}}$ is the ratio of specific heats, where $c_{\mathrm{p}}$ is the specific heat at a constant pressure and $c_{\mathrm{V}}$ is the specific heat at a constant volume. The constant parameter $v$ is a modified inverse Eckert number and describes the ratio of kinetic energy and enthalpy (Kay \& Nedderman 1985, p. 255). The last two constants $\hat{\delta}$ and $\hat{\Omega}$ describe aspects of the Coriolis force due to the rotation of Saturn. A definition sketch of the dimensionless computational $\delta$-plane is given in Fig. 3.

The velocity vector is $\boldsymbol{v}=u \boldsymbol{i}+v \boldsymbol{j}$, where $u$ and $v$ are the respective $x$ - and $y$-directed velocity components with respect to the coordinate geometry in Fig. 2. The governing equations are well known and will only be discussed briefly. The mass continuity equation is

$\frac{\partial \rho}{\partial t}+u \frac{\partial \rho}{\partial x}+v \frac{\partial \rho}{\partial y}+\rho\left(\frac{\partial u}{\partial x}+\frac{\partial v}{\partial y}\right)=0$.

The respective momentum equations for the velocity components are

$\frac{\partial u}{\partial t}+u \frac{\partial u}{\partial x}+v \frac{\partial u}{\partial y}-\hat{f} v+\frac{v}{\rho} \frac{\partial p}{\partial x}=0$,

$\frac{\partial v}{\partial t}+u \frac{\partial v}{\partial x}+v \frac{\partial v}{\partial y}+\hat{f} u+\frac{v}{\rho} \frac{\partial p}{\partial y}=0$,

where $\hat{f}$ is the dimensionless Coriolis parameter given by

$\hat{f}=2 \hat{\Omega}-\hat{\delta}\left(x^{2}+y^{2}\right)$.
As a result of assuming no addition of heat by external sources, the governing energy equation takes the form

$\frac{\partial T}{\partial t}+u \frac{\partial T}{\partial x}+v \frac{\partial T}{\partial y}+(\gamma-1) \frac{p}{\rho}\left(\frac{\partial u}{\partial x}+\frac{\partial v}{\partial y}\right)=0$.

This paper will look at ideal fluids that are weakly compressible, so that density $\rho$ is variable. An ideal fluid must obey the equation of state that takes the dimensionless form

$p=\rho T$

The dimensionless equation of state (11) is transformed by taking the time derivative, so that

$$
\begin{aligned}
\frac{\partial p}{\partial t}= & -T\left[u \frac{\partial \rho}{\partial x}+v \frac{\partial \rho}{\partial y}+\rho\left(\frac{\partial u}{\partial x}+\frac{\partial v}{\partial y}\right)\right] \\
& -\rho\left[u \frac{\partial T}{\partial x}+v \frac{\partial T}{\partial y}+(\gamma-1) \frac{p}{\rho}\left(\frac{\partial u}{\partial x}+\frac{\partial v}{\partial y}\right)\right],
\end{aligned}
$$

after use of (6) and (10).

Time independent steady state background flow behaviour is used to determine the boundary conditions. It will be assumed that the atmospheric flow is geostrophic and that the atmosphere is isopycnal. The Hexagon itself is embedded within an eastward circumpolar jet spanning the latitudes $74^{\circ}-78^{\circ}$, with peak velocity at $76^{\circ}$. Godfrey (1988) calculated the peak velocity to be approximately $100 \mathrm{~m} \mathrm{~s}^{-1}$, Baines et al. (2009) suggest it could be as high as $125 \mathrm{~m} \mathrm{~s}^{-1}$ and Antuñano et al. (2015) indicate that it is about $119 \mathrm{~m} \mathrm{~s}^{-1}$. The velocity drops significantly to less than $20 \mathrm{~m} \mathrm{~s}^{-1}$ at the boundary latitudes of the Hexagon. At the approximate latitude of $69^{\circ}$ the velocity is about $-20 \mathrm{~m} \mathrm{~s}^{-1}$, indicating a moderate westward jet to the south. These extremes of the velocity profile are paramount when deciding on the initial velocities of the numerical simulations. The velocity profile given by Godfrey (1988, fig. 4) will be the basis for the time independent steady background flow component of the non-linear representations given later. Due to the scaling of the problem, $1^{\circ}$ in latitude is approximately equal to a dimensionless distance of 0.1 and the background flow components take the form

$$
\begin{aligned}
u_{\mathrm{b}}(x, y)= & -C_{0} y \exp \left[-C_{1}\left(\sqrt{x^{2}+y^{2}}-1.4\right)^{2}\right] \\
& +C_{2} y \exp \left[-C_{3}\left(\sqrt{x^{2}+y^{2}}-2.1\right)^{2}\right], \\
v_{\mathrm{b}}(x, y)= & C_{0} x \exp \left[-C_{1}\left(\sqrt{x^{2}+y^{2}}-1.4\right)^{2}\right] \\
& -C_{2} x \exp \left[-C_{3}\left(\sqrt{x^{2}+y^{2}}-2.1\right)^{2}\right] .
\end{aligned}
$$

The constants 1.4 and 2.1 in the velocity representations (13) and (14) are the dimensionless distance from the origin of the $\delta$-plane to the latitude where the eastward and westward jets are at their maximum flow rates.

The flow region of interest is between latitudes $66^{\circ}$ and $80^{\circ}$ where the eastward and westward velocities are extremized around the hexagonal feature. Outside of this region, the background velocity will take the value of zero. A curve-fitting procedure was used to determine the values of the constants $C_{0}, C_{1}, C_{2}$ and $C_{3}$ so that the background flow had similar characteristics to the zonal velocity profile published by Godfrey (1988) over the flow region of interest. It was found that $C_{0} \approx 0.7, C_{1}=40, C_{2} \approx 0.1$ and $C_{3}=20$. The constants $C_{0}$ and $C_{2}$ are effective amplitude values for the exponential terms and the constants $C_{1}$ and $C_{3}$ are just dilation 


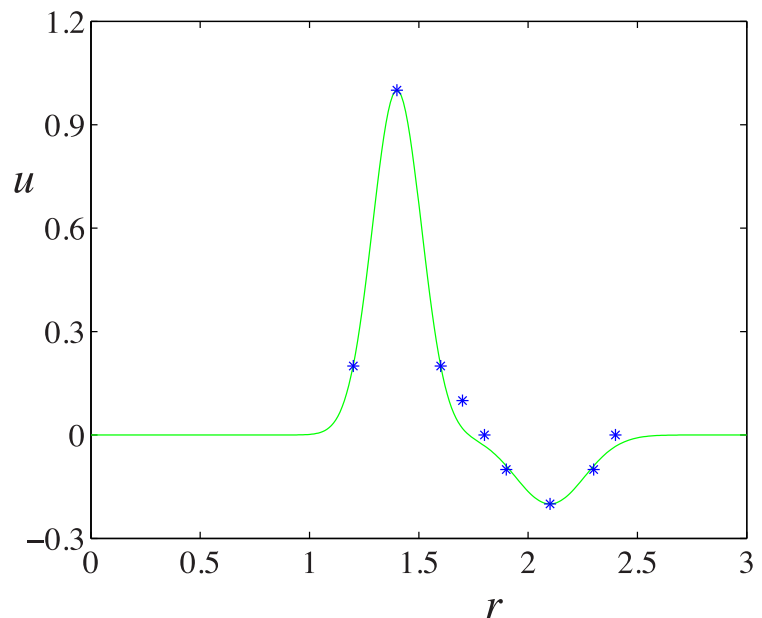

Figure 4. Curve-fitted zonal velocity profile. The blue crosses are values interpreted from the velocity profile published by Godfrey (1988) and expressed in dimensionless form.

factors of the peaks in the exponential representations. Fig. 4 shows a plot of the curve-fitted representation of the zonal velocity, with the blue crosses indicating values interpreted from the zonal velocity profile published by Godfrey (1988) in dimensionless form, with $u$ representing the zonal velocity and $r$ is the radius from the pole.

For the isopycnal atmosphere, the constant density in dimensionless variables is simply

$\rho_{\mathrm{b}}(x, y)=1$.

The equation of state (11) must be satisfied and thus it follows that the background pressure $p_{\mathrm{b}}(x, y)$ and temperature $T_{\mathrm{b}}(x, y)$ are initially equal. The material derivative terms of the momentum equations (7) and (8) are ignored under the geostrophic assumption so that they can be re-arranged and simplify, approximately, to

$\frac{\partial p_{\mathrm{b}}(x, y)}{\partial y}=-\frac{u_{\mathrm{b}}(x, y) \hat{f}}{v}$

$\frac{\partial p_{\mathrm{b}}(x, y)}{\partial x}=\frac{v_{\mathrm{b}}(x, y) \hat{f}}{v}$.

To get a representation for the background pressure, equation (16) is integrated with respect to $y$, the result is then differentiated with respect to $x$ and compared to equation (17). Thus, the background pressure (and background temperature) has the form

$$
\begin{aligned}
p_{\mathrm{b}}(x, y)= & \frac{C_{0}}{v} \int_{0}^{\xi} r \hat{f} \exp \left[-C_{1}(r-1.4)^{2}\right] \mathrm{d} r \\
& -\frac{C_{2}}{v} \int_{0}^{\xi} r \hat{f} \exp \left[-C_{3}(r-2.1)^{2}\right] \mathrm{d} r+\mathcal{K} \\
= & T_{\mathrm{b}}(x, y) .
\end{aligned}
$$

Here, $r=\sqrt{x^{2}+y^{2}}=\xi$ and the constant $\mathcal{K}$ is chosen such that the pressure and temperature take the values $p=1$ and $T=1$, respectively, on the boundaries of the $\delta$-plane.

It has been suggested by Allison et al. (1990) that the hexagonal structure over the pole of Saturn is the result of at least one perturbing anticyclone. This claim by Allison et al. (1990) appears not to have been investigated thoroughly in the literature. For this reason, the effects of perturbing anticyclone(s) will be simulated to infer whether or not anticyclones could be a possible trigger mechanism for the formation of the North Polar Hexagon on Saturn. The perturbing anticyclones are modelled using high-pressure systems in the form

$$
\begin{aligned}
p_{\mathrm{p}}(x, y)= & \mu_{1} \exp \left[\frac{-\left((x-M)^{2}+(y-M)^{2}\right)}{\sigma_{1}^{2}}\right] \\
& +\mu_{2} \exp \left[\frac{-\left((x+M)^{2}+(y+M)^{2}\right)}{\sigma_{2}^{2}}\right] \\
= & T_{\mathrm{p}}(x, y) .
\end{aligned}
$$

The rise in pressure is given by the values of $\mu_{1}, \mu_{2}$ and the radii of the anticyclones are $\sigma_{1}, \sigma_{2}$. For convenience if $\mu_{1}=m u_{2}$ the pressure rise will be denoted $\mu$, similarly if $\sigma_{1}=\sigma_{2}$ the radii will be specified as $\sigma$. The initial locations of the anticyclones are the Cartesian coordinates $\pm(M, M)$. A direct result of the equation of state (11) and the isopycnal assumption is that the perturbing density must be

$\rho_{\mathrm{p}}(x, y)=1$.

The velocity components of the atmospheric flow induced by the anticyclones are

$$
\begin{aligned}
& u_{\mathrm{p}}(x, y)=-\frac{v}{\hat{f}} \frac{\partial p_{\mathrm{p}}(x, y)}{\partial y}, \\
& v_{\mathrm{p}}(x, y)=\frac{v}{\hat{f}} \frac{\partial p_{\mathrm{b}}(x, y)}{\partial x} .
\end{aligned}
$$

\section{THE SPECTRAL SOLUTION METHOD}

This section discusses the numerical techniques used in order to solve for the five dependent variables, pressure $p$, density $\rho$, temperature $T$ and the $x$ - and $y$-directed velocity components $u$ and $v$. Spectral solutions using both Dirichlet and Robin boundary conditions are sought and a comparison of both approaches is discussed in the results section. The following outlines the derivation of the Dirichlet boundary value problem and the earlier paper by Cosgrove $\&$ Forbes (2017) gives a derivation for the corresponding similar Robin boundary value problem.

The state variables are given the value one on the boundaries of the $\delta$-plane and the velocity components are zero there. This leads to the spectral representation

$p(x, y, t)=1+\sum_{m=1}^{\infty} \sum_{n=1}^{\infty} P_{m n}(t) F_{m}(x) G_{n}(y)$,

$\rho(x, y, t)=1+\sum_{m=1}^{\infty} \sum_{n=1}^{\infty} R_{m n}(t) F_{m}(x) G_{n}(y)$,

$T(x, y, t)=1+\sum_{m=1}^{\infty} \sum_{n=1}^{\infty} T_{m n}(t) F_{m}(x) G_{n}(y)$,

$u(x, y, t)=\sum_{m=1}^{\infty} \sum_{n=1}^{\infty} A_{m n}(t) F_{m}(x) G_{n}(y)$,

$v(x, y, t)=\sum_{m=1}^{\infty} \sum_{n=1}^{\infty} B_{m n}(t) F_{m}(x) G_{n}(y)$.

The time-dependent Fourier coefficients $P_{m n}(t), R_{m n}(t), T_{m n}(t)$, $A_{m n}(t)$ and $B_{m n}(t)$ are to be determined. 
The basis functions $F_{m}(x)$ and $G_{n}(y)$ are chosen so that the Dirichlet boundary conditions that are imposed on the unsteady timedependent components take the form

$p( \pm \lambda, y, t)=0 ; p(x, \pm \omega, t)=0$

for the pressure and similarly for the remaining series terms. This leads to sinusoidal basis functions

$F_{m}(x)=\sin \left(\frac{m \pi(x+\lambda)}{2 \lambda}\right)$

$G_{n}(y)=\sin \left(\frac{n \pi(y+\omega)}{2 \omega}\right)$.

The governing equations (6)-(10) and (12) are used to evaluate the time-dependent coefficients $P_{m n}(t), R_{m n}(t), T_{m n}(t), A_{m n}(t)$ and $B_{m n}(t)$ by isolating the time-dependent and spatial components of each governing equation. For example, the Fourier coefficients $A_{m n}(t)$ used in the $x$-directed velocity component are determined by using the non-linear representation (26) and the $x$-directed momentum equation (7) together to give the expression

$\frac{\partial u}{\partial t}=\sum_{m=1}^{\infty} \sum_{n=1}^{\infty} A_{m n}^{\prime}(t) F_{m}(x) G_{n}(y)=-F_{A}(x, y, t)$,

where

$F_{A}(x, y, t)=u \frac{\partial u}{\partial x}+v \frac{\partial u}{\partial y}-\hat{f} v+\frac{v}{\rho} \frac{\partial p}{\partial x}$.

The function $F_{A}(x, y, t)$ represents the spatially varying terms in the $x$-directed momentum equation (7). Spectral decomposition is used to develop a system of ordinary differential equations for the Fourier coefficients $A_{m n}(t)$. This is achieved by multiplying by basis functions $F_{k}(x), G_{l}(y)$ and integrating over the region of the $\delta$-plane giving

$A_{k l}^{\prime}(t)=\frac{-1}{\lambda \omega} \int_{-\lambda}^{\lambda} \int_{-\omega}^{\omega} F_{A}(x, y, t) F_{k}(x) G_{l}(y) \mathrm{d} y \mathrm{~d} x$
for $\mathrm{k}=1,2, \ldots, \mathrm{M}$, and $, \mathrm{l}=1,2, \ldots, \mathrm{N}$.

The Fourier coefficients $P_{m n}(t), R_{m n}(t), T_{m n}(t)$ and $B_{m n}(t)$ are derived in the exact same manner and will not be stated here.

\section{PRESENTATION OF RESULTS}

Allison et al. (1990) proposed that the hexagonal feature centred at the north pole of Saturn is a Rossby wave which may have been caused by at least one perturbing anticyclone to the south. Although there are no such anticyclones currently observed in the vicinity of the Polar Hexagon, this section will outline whether or not it is possible for a hexagonal feature to be formed by an anticyclone impinging on the boundary of a larger cyclone. The results that follow are obtained through numerical simulations using parameter values close to those observed on Saturn with basic atmospheric assumptions. The majority of results outlined in this section will be presented in the form of pressure contour charts. Solutions will be given for single and binary perturbing anticyclones. The variation in the location, radius and pressure differential of the perturbing anticyclones will also be considered.

The pressure scale is $p_{0}=\rho_{0} R T_{0}=1 \mathrm{bar}=10^{5} \mathrm{~Pa}$. At this pressure, the Saturnian atmosphere has a temperature of approximately $134^{\circ} \mathrm{K}$ (Williams 2015) and so the scale temperature $T_{0}=134^{\circ} \mathrm{K}$ and scale density of $\rho_{0}=0.186 \mathrm{~kg} \mathrm{~m}^{-3}$ will be assumed. The dimensionless half-plane width and breadth are $\lambda=\omega=6$, so that the $\delta$-plane region is $-6 \leq x \leq 6,-6 \leq y \leq 6$. These values are

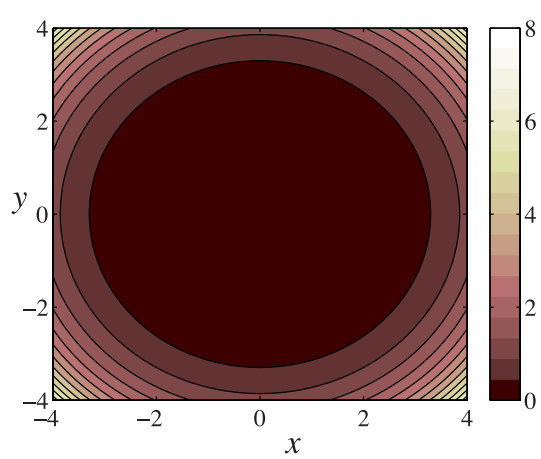

Figure 5. Contour diagram of the approximation error. The scale gives the percentage relative error (33) in the $\delta$-plane assumption.

chosen so that there is ample space between where the actual flow of interest occurs and the location of the artificial $\delta$-plane boundaries. In the results that follow the flow feature of concern is more or less bounded by the region $-\frac{5}{2}<x<\frac{5}{2},-\frac{5}{2}<y<\frac{5}{2}$ where there is much less than 1 percent difference between Coriolis parameter value given by (1) and the associated $\delta$-plane value (9). This qualitative value is determined using the error approximation function

$E r(x, y)=\frac{2 \hat{\Omega} \sin \phi-2 \hat{\Omega}+\hat{\delta}\left(x^{2}+y^{2}\right)}{2 \Omega \sin \phi} \times 100$.

The contours of the error as given by (33) are shown in Fig. 5.

The background flow will be perturbed with either a single anticyclone or a binary anticyclone system as given in equation (19); setting $\mu_{2}=0$ in this equation produces a single anticyclone. The initial eye locations of the perturbing system(s) will be $(x, y)=(M, M)$ or $(x, y)= \pm(M, M)$, hereafter denoted $M$. This geometry is chosen so that the possible interference caused by reflections off the artificial boundaries when using spectral methods takes its maximum time to impact on the numerical solution and thus reliable, informative solutions can be obtained. The figures throughout this section will be magnified over a region smaller than the computational domain of $\lambda=\omega=6$, so that the intricacies of the flow can be more readily shown. This section will attempt to answer the following questions: (1) Is it possible for perturbing anticyclone(s) to produce a hexagonal structure over the pole of Saturn? (2) What effect does the location, radius and pressure differential have on the formation of a hexagon?

The types of background flow coupled with the perturbation flow have varying degrees of influence on the evolving flow pattern. To get an overall feel for what is happening, quiver plots of the velocity field using the horizontal velocity representations (13), (21) and the vertical velocity components (14), (22) are shown in Fig. 6 and confirm the eastward and westward circumpolar jets as indicated by the respective anticlockwise and clockwise directional quivers. As expected there is an annulus of stagnation flow between both jets. The perturbing quiver plots in Figs 6(a) and (b) clearly show the anticyclonic rotation of the high-pressure regions. The perturbing anticyclones also interrupt the stagnation ring, particularly in this case when the perturbations are in close proximity to the easterly jet and thus impinge on the flow.

The first image of the North Polar Hexagon (Godfrey 1988) showed that there was at least one anticyclone to the south of the hexagonal structure. In particular, it was proposed that the Hexagon may be the result of at least one perturbing anticyclone (Allison et al. 1990). The pressure differential in such an anticyclone has a 
(a)

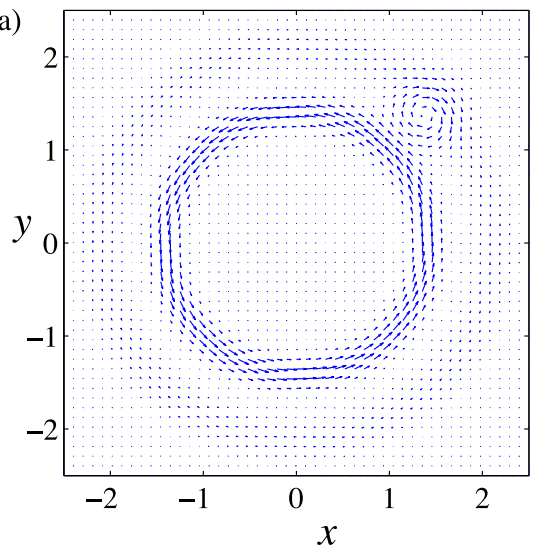

(b)

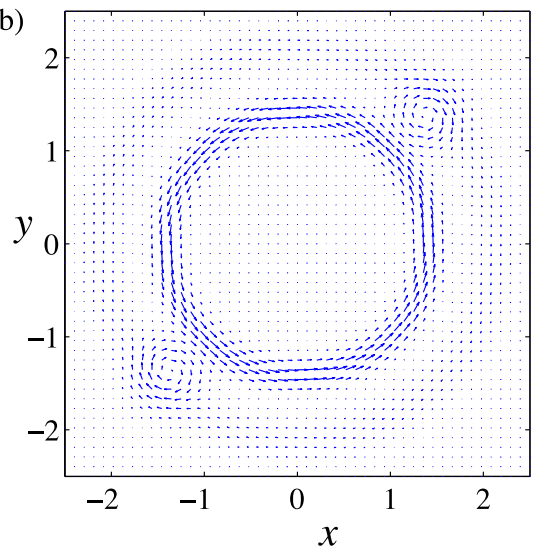

Figure 6. Quiver plots of the initial background velocity with perturbation from (a) a single anticyclone and (b) two anticyclones.

major impact on the flow strength around the eastward jet. Figs 7(a) and (b) show the quivers of the initial velocity field for the respective pressure differential of $\mu=0.1$ and $\mu=0.2$. In these figures, the eyes of the anticyclones are located at $M=1.5$ with radius $\sigma=0.4$, and although generated over the entire computational domain, both figures are cropped so that the flow pattern and strength are emphasized. The larger the pressure differential value $\mu$, the more intense the perturbing system as indicated by the large-magnitude quivers in the top-right corner of Fig. 7(b). An additional result made clear in these two diagrams is the pin-wheel effect occurring in the interfacial region between the two opposing flow directions. This results in fluid being ejected tangentially towards the top left of the $\delta$-plane.

The initial conditions for the polar flow on the $\delta$-plane were determined by making the atmosphere initially isopycnal so that the density is just the background density $\rho=1$ and is achieved by setting $R_{m n}(0)=0$ in equation (24). A direct consequence of the initial isopycnal atmosphere is that the initial pressure and temperature are identically defined as

$p(x, y, 0)=1+H(x, y)=T(x, y, 0)$,

where $H(x, y)$ is conveniently defined as a slightly modified sum of the background (18) and perturbation (19) components for the pressure and temperature and is of the form

$$
\begin{aligned}
H(x, y)= & \frac{C_{0}}{v} \int_{0}^{\xi} r f \exp \left[-C_{1}(r-1.4)^{2}\right] \mathrm{d} r \\
& -\frac{C_{2}}{v} \int_{0}^{\xi} r f \exp \left[-C_{3}(r-2.1)^{2}\right] \mathrm{d} r
\end{aligned}
$$
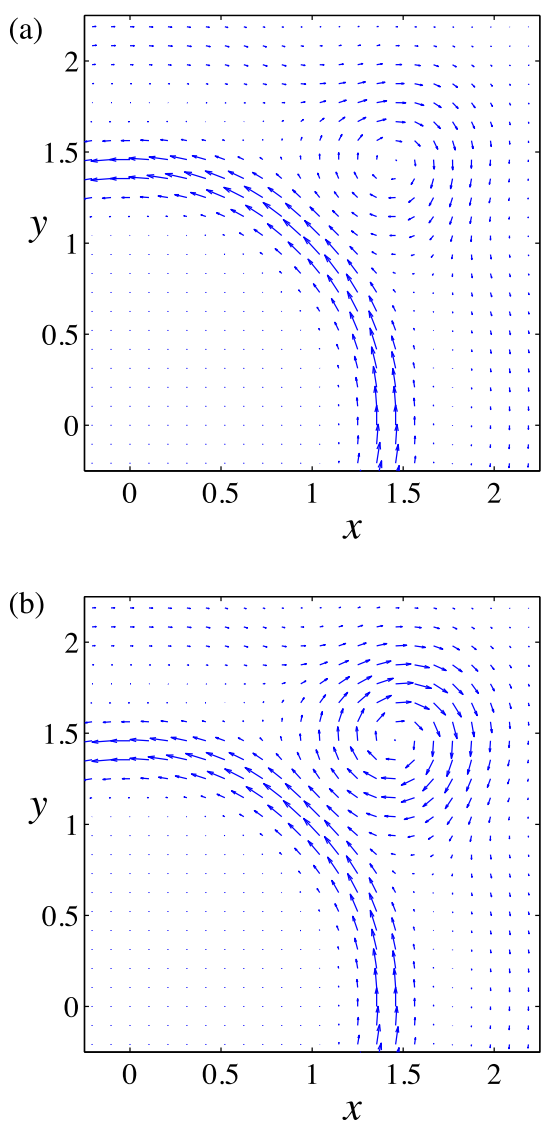

Figure 7. Quiver plots of the initial background velocity with perturbing anticyclone of pressure differential (a) $\mu=0.1$ and (b) $\mu=0.2$.

$$
\begin{aligned}
& +\mu_{1} \exp \left[\frac{-\left((x-M)^{2}+(y-M)^{2}\right)}{\sigma_{1}^{2}}\right] \\
& +\mu_{2} \exp \left[\frac{-\left((x+M)^{2}+(y+M)^{2}\right)}{\sigma_{2}^{2}}\right]+\mathcal{S} .
\end{aligned}
$$

The constant $\mathcal{S}$ is chosen so that $H(x, y)=0$ on the boundary of the $\delta$-plane and $r$ and $\xi$ are as defined previously. The initial Fourier coefficients $P_{m n}(0)$ and $T_{m n}(0)$ for the pressure and temperature functions are found using spectral decomposition. Thus

$$
\begin{aligned}
P_{m n}(0) & =\frac{1}{\lambda \omega} \int_{-\lambda}^{\lambda} \int_{-\omega}^{\omega} H(x, y) F_{k}(x) G_{l}(y) \mathrm{d} y \mathrm{~d} x \\
& =T_{m n}(0) .
\end{aligned}
$$

The initial Fourier coefficients for velocity components $A_{m n}(0)$ and $B_{m n}(0)$ are similarly found. For $A_{m n}(0)$, the function $H(x, y)$ in the integrand of equation (35) is replaced with the sum of the $x$-directed background flow (13) and perturbation flow (21). Likewise, the sum of the $y$-directed components (14) and (22) are used to determine $B_{m n}(0)$. The quadratures are performed using the Gaussian integration routine provided by von Winckel (2004).

A three-dimensional plot of the initial pressure and temperature for the unperturbed and perturbed flows is shown in Figs 8(a) and (b). In these figures, the unperturbed flow induces values of dimensionless pressure in the approximate interval $0.88<p<1.04$, with $p=1$ on the boundaries. The easterly circumpolar jet produces a region of low pressure and the westerly flow creates a high-pressure annulus. The perturbing anticyclones are located at $M=1.4$ with 
(a)

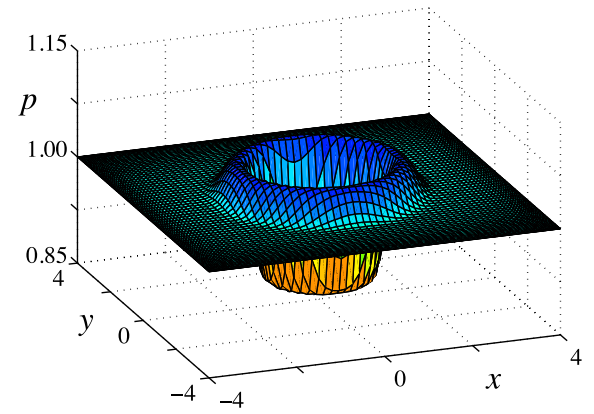

(b)

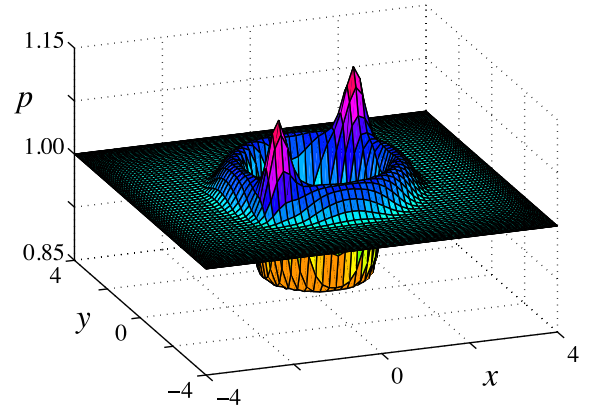

Figure 8. Sketch of 2 three-dimensional initial pressure and temperature profiles for (a) unperturbed flow and (b) perturbed flow.

radius $\sigma=0.3$ and pressure differential $\mu=0.1$, and thus increase the pressure to $p \approx 1.14$ at the eye.

The results of perturbing the underlying circumpolar jets with a single anticyclone of radius $\sigma=0.4$ located initially at $M=1.5$ are shown in Fig. 9, at time $t=1.6$. When the anticyclone has strength $\mu=0.1$ there is no significant change in the initial circular shape of the pressure system, which can be seen in Fig. 9(a). However, when the pressure differential of the anticyclone is increased to $\mu=0.2$, as illustrated in Fig. 9(b), there is evidence of the anticyclone influencing the flow, in particular, the creation of a straight edge between the two opposing systems. Furthermore, there are two rounded vertices, one at each end of the edge forming what could be interpreted as the beginning of a hexagonal portion of a trapezoid. Our simulations using one anticyclone failed to produce a hexagonal feature, but instead, generated a circular-shaped region but with one flattened side, as shown in Fig. 9(b).

If one perturbing anticyclone could produce a trapezoid type structure as indicated in Fig. 9(b), then two symmetrically placed anticyclones may just produce what is proposed. Fig. 10 is a timelapse illustration of the influence two perturbing anticyclones have on the pressure structure over the polar region. The pair of anticyclones is modelled using equation (19). The initial placement of the anticyclones is $M=1.5$ with $\sigma=0.4$ and $\mu_{1}=\mu_{2}=0.2$. Hereafter, if the pressure change is equal in both anticyclones $\left(\mu_{1}=\mu_{2}\right)$, then the pressure change will be expressed as $\mu$ alone. For the single perturbing anticyclone with the same characteristics as the pair in Fig. 10, the trapezoidal formation was first observed at time $t=1.6$. Thus, Fig. 10(a) shows the contour map of the binary perturbation at the same time $t=1.6$. At this time, the hexagonal structure can already be seen. Figs 10(b)-(d) show the evolution of the pressure at the times $t=1.8,2.0$ and 2.2, respectively. As the time increases to $t=2.0$ the hexagon is at its most regular. Although the feature is still prominent at the later time $t=2.2$, there are ripples forming along the edges non-adjacent to the anticyclones. At time $t=3.0$,
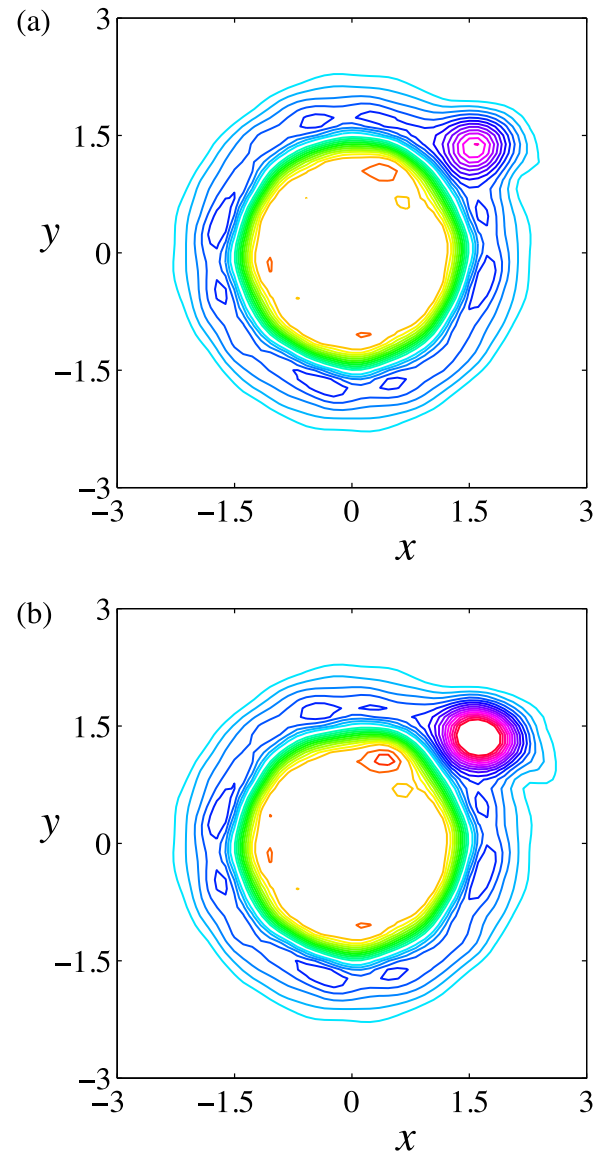

Figure 9. Pressure contour plots with single anticyclone located originally at $M=1.5$ of radius $\sigma=0.4$ at time $t=1.6$ with pressure differential (a) $\mu=0.1$ and (b) $\mu=0.2$.

the hexagonal structure is still evident; however, there are clear indentations along the edges to the west of the anticyclones indicating the structure is beginning to collapse. Due to the short lifespan of the hexagonal structures, it is hard to determine for how long the hexagon is approximately stationary. If it is stationary over the approximate dimensionless time $1.6<t<4.0$, this would correspond to a time interval of approximately $3 \mathrm{~d}$. This is not the case for the perturbing anticyclones; the location of the anticyclones is within the westward jet and thus there is clear movement to their west.

It is interesting to note the closed elliptical contours aligned centrally along the outer edges of the hexagonal structure. These are isolated regions of higher pressure in the form of smaller, less intense anticyclones, which is consistent with what Godfrey (1988) observed through streamline maps.

It has now been shown that a hexagonal feature, although transient, can be formed through anticyclones impinging on the boundary of a circumpolar jet and thus the results shown in Fig. 10 can be used as a baseline to determine the effects that the initial location $M$, radius $\sigma$ and pressure differential $\mu$ have on the development of a hexagonal structure. The location of the initial anticyclones needs to be within a close enough proximity to influence the flow associated with the eastward jet. Moving the location of the anticyclones closer to the jet, so that $M=1.4$ and keeping all other baseline parameters identical, inhibits the formation of the furthest vertex from the anticyclones being formed. This is depicted in Fig. 11(a) and shows the most regular hexagonal type feature that occurred 
(a)

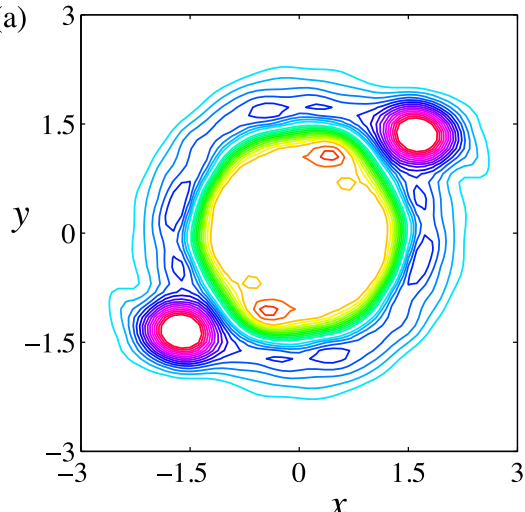

(b)

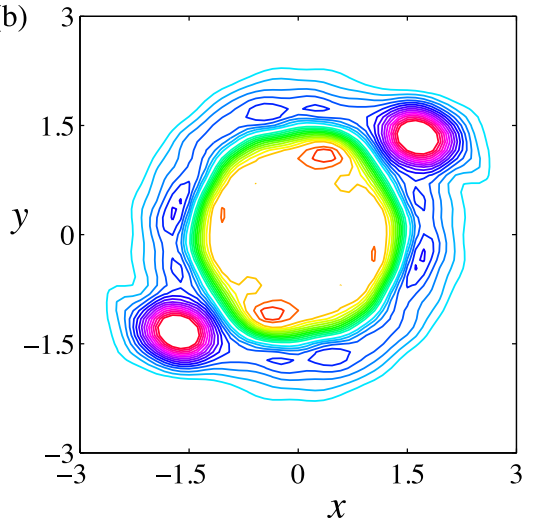

(c)

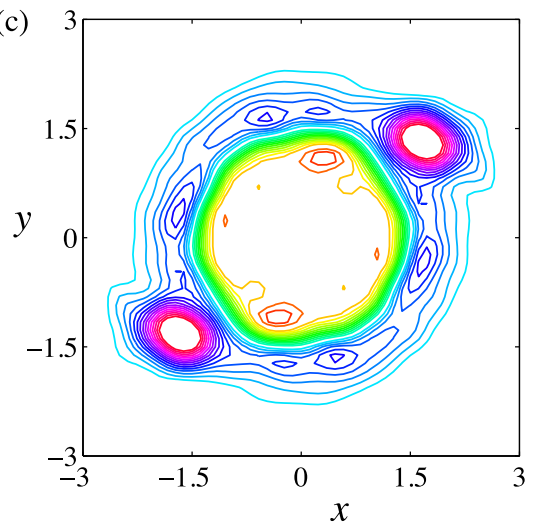

(d)

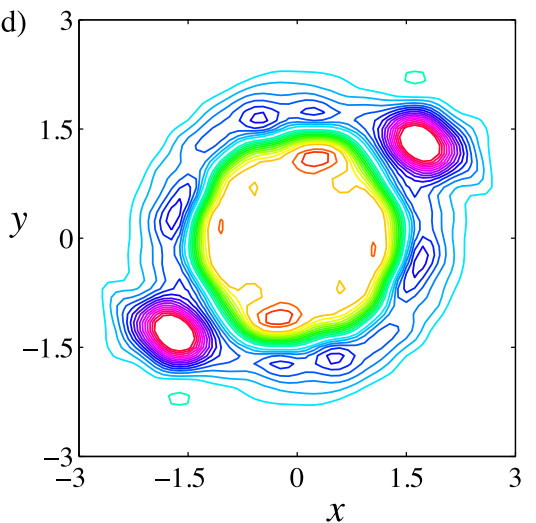

Figure 10. Pressure contour plots with two perturbing anticyclones located originally at $M=1.5$ of radius $\sigma=0.4$ with pressure differential $\mu=0.2$ at times (a) $t=1.6$, (b) $t=1.8$, (c) $t=2.0$ and (d) $t=2.2$.
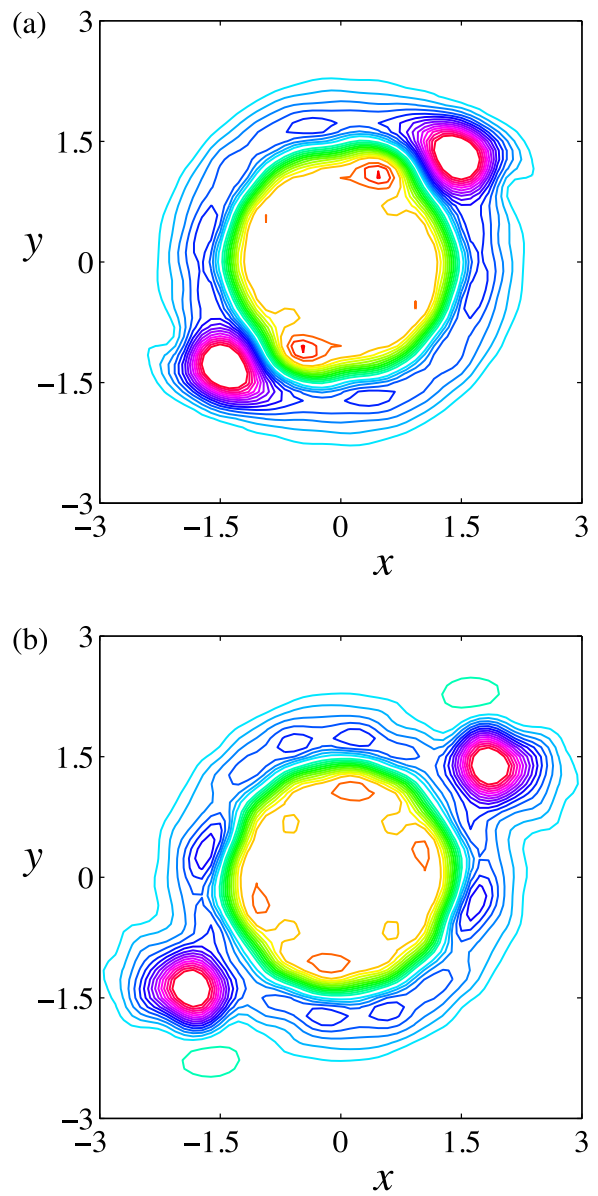

Figure 11. Pressure contour plots with two perturbing anticyclones of radius $\sigma=0.4$ for (a) $M=1.4, t=1.2$ and (b) $M=1.6, t=2.4$.

with $M=1.4$. As would be expected, the hexagonal type structure formed at the earlier time of $t=1.2$ compared to the baseline time of $t=2.0$. Although there is a hexagonal structure, it is less defined than that of Fig. 10. Conversely moving the initial eye out to $M=1.6$ a hexagonal structure is never formed. However, there is a slight vertex being formed to the west of each anticyclone which may be the results of the pin-wheel effect; this is shown in Fig. 11(b) at the later time $t=2.4$.

Decreasing the magnitude of the pressure differential plays a significant role in the formation of a hexagonal structure. Fig. 12(a) is the contour diagram at time $t=2.0$ using the baseline parameters except that the pressure differential is now $\mu=0.1$. At this time there is no significant hexagonal formation developing, whereas for the baseline simulation with $\mu=0.2$ the hexagon was at its most regular. However, with this decrease in pressure differential a hexagon is produced at time $t=2.0$ when the anticyclone is initially closer to the easterly jet. This is illustrated in Fig. 12(b) which has $\mu=0.1$ and $M=1.4$.

Although not shown here, when the pressure differential $\mu$ is unchanged, the smaller the radius of the anticyclone, the greater the internal flow velocity. Fig. 13 shows the effect of changing the radius of the anticyclones as compared to the baseline simulation. The respective pressure contour plots at time $t=2.0$ with smaller radius $\sigma=0.3$ and larger radius $\sigma=0.5$ are shown in Figs 13(a) and (b). There is no indication of a hexagon being formed when $\sigma=0.3$. This can be attributed to the fact that the anticyclones are sufficiently 

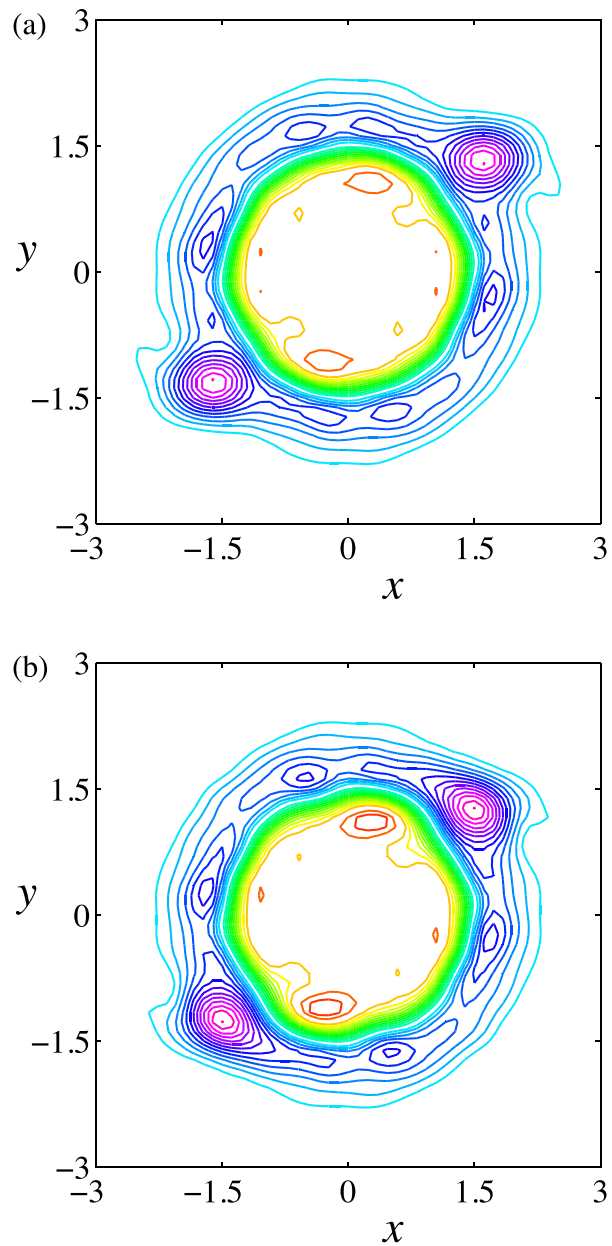

Figure 12. Pressure contour plots with two perturbing anticyclones of radius $\sigma=0.4$, pressure differential $\mu=0.1$ at time $t=2.0$ for location (a) $M=1.5$ and (b) $M=1.4$

separated from the eastward jet, so that the greater internal velocity has no influence on the overall polar flow. The converse is true when the radius is $\sigma=0.5$. The internal velocities are smaller in this case compared to when the radius is $\sigma=0.3$, but due to the initial location being identical, the larger radius anticyclones have impact on the polar flow, and thus on the deformed hexagonal structure. For the larger radius $\sigma=0.5$, the hexagonal feature was most regular at time $t=1.5$.

Morales-Juberias et al. (2011) showed that stable polygons can be formed by 'vortex streets' with cyclonic and anticyclonic vortices aligning themselves on the pole and equator sides of the eastward circumpolar jet, respectively. However, they suggest that the vortex street model and the observed wind profile are incompatible. The governing equations used in our simulations are not formulated in terms of potential vorticity. However, as a bi-product of calculating the velocity components forward in time, the vorticity can be determined at any given time. Vorticity is a way to describe the local rotation of a fluid about some point and is simply given by

$\zeta=\frac{\partial v}{\partial x}-\frac{\partial u}{\partial y}$.

The contour plots shown in Fig. 14 are of the local vorticity of the baseline perturbing system in Fig. 10. The vorticity (37) is computed by direct differentiation of the Fourier series (26), (27) at the desired time. As expected the perturbing anticyclones
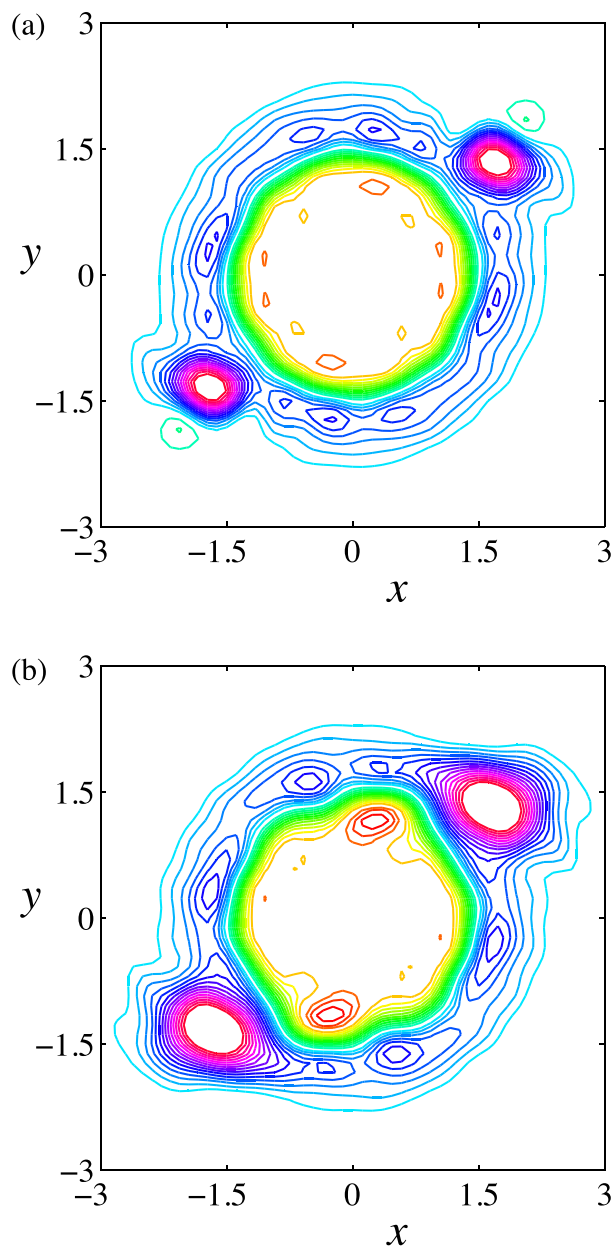

Figure 13. Pressure contour plots with two perturbing anticyclones located originally at $M=1.5$ with pressure differential $\mu=0.2$ at time $t=2.0$, for radius (a) $\sigma=0.3$ and (b) $\sigma=0.5$.

have negative vorticity, in the interval $-9<\zeta<-1$, and the inner hexagonal region is the perturbed northernmost section of the eastward jet and has positive vorticity ranging over $1<\zeta<5$. For the outer regions of the $\delta$-plane, where the quiver plots indicate that there is negligible flow, the vorticity is similarly zero. There is remarkable agreement in the shape of the vorticity contours to those of the pressure contours at the same times.

It is shown in Fig. 8 that the unforced circumpolar jets have pressure ranging from $p=0.88$ to $p=1.04$. The intermediate pressure value $p=0.96$ is a good indicator of the crossover from positive to negative vorticity regions, as well as the defining pressure contour of the hexagonal structure. This fact is confirmed in Fig. 15 and shows the pressure and vorticity contours in the respective subfigures (a) and (b) at time $t=0.19$ for the baseline configuration $M=1.5, \sigma=0.4$ and $\mu=0.2$, combined with the interfacial pressure contour $p=0.96$ in black.

In trying to keep the simulations aligned with what was first observed by Godfrey (1988), the parameter space for the single perturbing anticyclone was kept to eye locations $M \in[1.4,1.5]$ and radii $\sigma=0.3$ and $\sigma=0.4$. It was evident from these simulations that a hexagonal structure was unlikely to be formed. However, the results obtained from the single anticyclone simulations indicated that it may be possible for a hexagonal feature to be formed by introducing a second symmetrically placed anticyclone. Table 1 
(a)

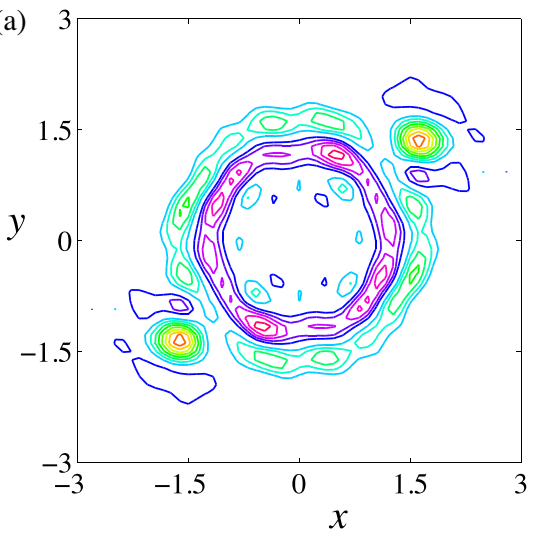

(b)

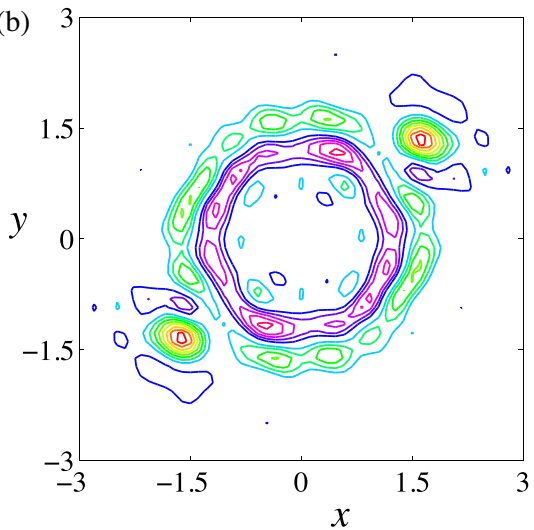

(c)

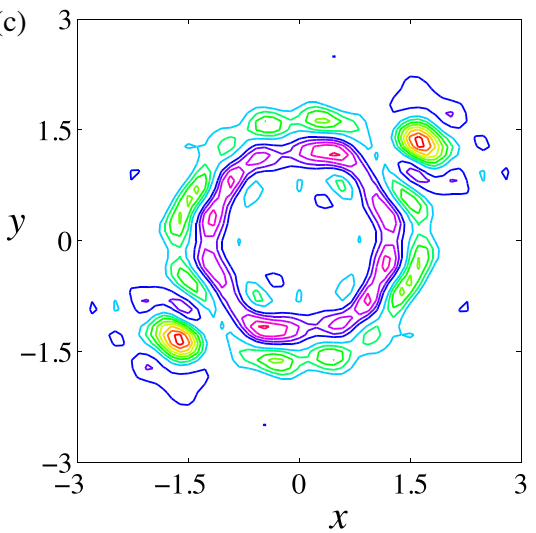

(d)

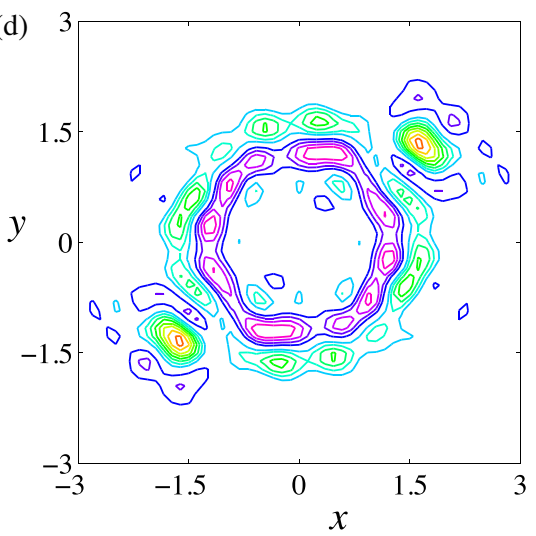

Figure 14. Vorticity contour plots with two perturbing anticyclones located originally at $M=1.5$ of radius $\sigma=0.4$ with pressure differential $\mu=0.2$ at times (a) $t=1.6$, (b) $t=1.8$, (c) $t=2.0$ and (d) $t=2.2$. (a)

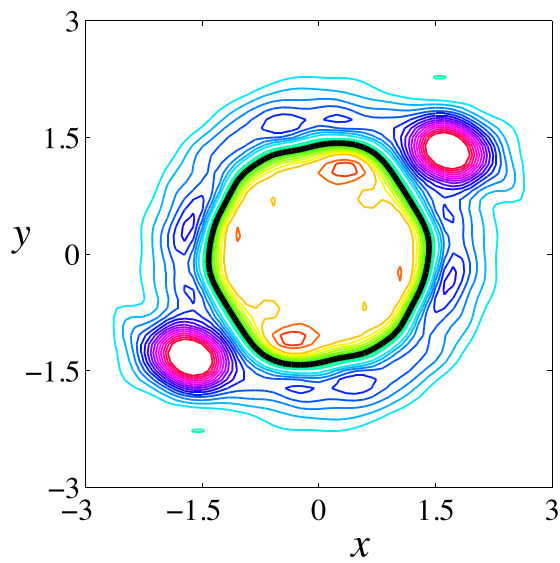

(b)

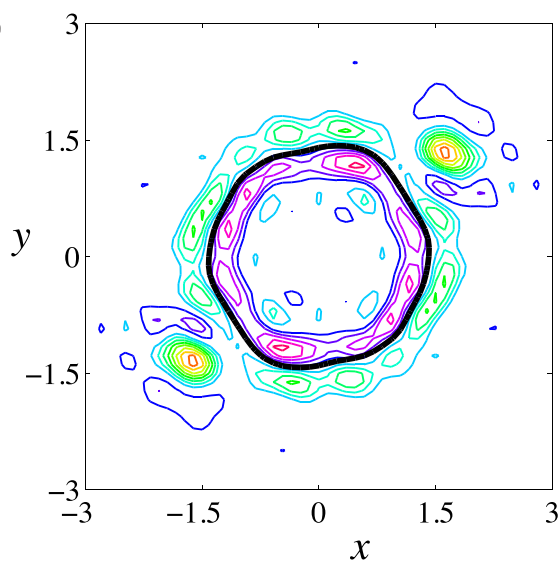

Figure 15. Contour plots with two perturbing anticyclones located originally at $M=1.5$ of radius $\sigma=0.4$ with pressure differential $\mu=0.2$ at time $t=1.9$ for (a) pressure and (b) vorticity. The thick black lines indicate the interfacial pressure contour $p=0.96$.

shows a list of all the space parameters investigated in this paper for two perturbing anticyclones, the asterisk indicates that a single anticyclone perturbation was also simulated. The fourth column of Table 1 indicates the lifespan of hexagonal structures that resulted. The lifespan of a hexagonal feature is given as the approximate time range from formation to dissipation.

The results above arise from perturbing an eastward circumpolar jet with anticyclones to the south and thus the problem is essentially an interacting atmospheric vortex problem. It has been shown previously by Cosgrove $\&$ Forbes (2017) that the results of simulating interacting atmospheric vortices using the $f$-plane approximation can be misleading due to false reflections off the artificial boundaries, especially when Dirichlet boundary conditions are involved. To overcome this phenomenon, Cosgrove \& Forbes (2017) used Robin boundary conditions to absorb and minimize the influence of such reflections. However, using the $\delta$-plane approximation, the numerical solutions obtained using Dirichlet and Robin boundary conditions are virtually identical, indicating that the $\delta$-plane approximation with its quadratic variation is capable of absorbing false reflections. An example of the similarity between the numerical solutions for the Dirichlet and Robin boundary value problems are shown in Figs 16(a) and (b), respectively.

It is clear from the smoothed zonal velocity profiles given by Godfrey (1988), Baines et al. (2009) and Antuñano et al. (2015) that there is considerable change in the magnitude of the observed maximum velocity within the hexagonal structure. The one constant 
Table 1. Shows all combinations of the simulation parameters investigated and lifespan of hexagonal structure.

\begin{tabular}{lccc}
\hline $\begin{array}{l}\text { Eye location } \\
M\end{array}$ & $\begin{array}{c}\text { Anticyclone } \\
\text { radii, } \sigma\end{array}$ & $\begin{array}{c}\text { Pressure } \\
\text { differential, } \mu\end{array}$ & Lifespan \\
\hline 1.3 & 0.3 & 0.1 & $1.6 \leq t \leq 1.8$ \\
1.3 & 0.3 & 0.2 & \\
1.3 & 0.4 & 0.05 & $2.0 \leq t \leq 2.3$ \\
1.3 & 0.4 & 0.1 & \\
1.3 & 0.4 & 0.2 & \\
$1.4^{*}$ & $0.3^{*}$ & $0.1^{*}$ & $1.5 \leq t \leq 1.7$ \\
$1.4^{*}$ & $0.3^{*}$ & $0.2^{*}$ & \\
$1.4^{*}$ & $0.3^{*}$ & $0.3^{*}$ & $1.4 \leq t \leq 2.2$ \\
$1.4^{*}$ & $0.4^{*}$ & $0.1^{*}$ & $1.2 \leq t \leq 1.7$ \\
$1.4^{*}$ & $0.4^{*}$ & $0.15^{*}$ & $1.7 \leq t \leq 2.2$ \\
$1.4^{*}$ & $0.4^{*}$ & $0.2^{*}$ & \\
$1.45^{*}$ & $0.4^{*}$ & $0.1^{*}$ & \\
$1.45^{*}$ & $0.4^{*}$ & $0.2^{*}$ & $1.1 \leq t \leq 1.8$ \\
$1.5^{*}$ & $0.3^{*}$ & $0.1^{*}$ & \\
$1.5^{*}$ & $0.3^{*}$ & $0.2^{*}$ & \\
$1.5^{*}$ & $0.4^{*}$ & $0.1^{*}$ & \\
$1.5^{*}$ & $0.4^{*}$ & $0.2^{*}$ & $1.4 \leq t \leq 3.0$ \\
$1.5^{*}$ & $0.4^{*}$ & 0.3 & \\
1.5 & 0.5 & 0.2 & \\
1.6 & 0.4 & 0.1 & \\
1.6 & 0.4 & 0.2 & \\
1.6 & 0.4 & 0.3 & \\
1.6 & 0.5 & 0.1 & \\
1.6 & 0.5 & 0.2 & \\
1.7 & 0.6 & 0.1 & \\
1.7 & 0.6 & & \\
\hline
\end{tabular}

feature of all three aforementioned velocity profiles is that the maximum velocity is observed at approximately $76^{\circ}$ latitude within the hexagonal region and steadily declines towards the outer boundaries of $74^{\circ}$ and $78^{\circ}$. Moreover, the most recent measurements reported by Antuñano et al. (2015) have the velocity within the Hexagon being in the range $104 \pm 15 \mathrm{~m} \mathrm{~s}^{-1}$. To get an idea of the effect of the velocity variation, Figs 17(a) and (b) show the respective pressure contour diagrams using the baseline parameters with a maximum velocity that is 75 per cent and 125 per cent of that used in the simulations above. The smaller velocity magnitude forms a more regular hexagonal feature but takes longer to develop; conversely, the hexagonal feature formed using the increased velocity evolves more quickly but is less regular.

The spectral representations have used $M=N=41$ Fourier coefficients with 161 sample points in each spatial variable. The results above have centred on pressure contour diagrams and to indicate the convergence of the numerical solution Fig. 18(a) shows the spectral representation of the initial pressure using equation (23) and Fig. 18(b) illustrates the initial 'true' closed form of the pressure given by the sum of the components (18) and (19). The only observable differences between the figures are the contour slivers in the westward jet at 3, 6, 9 and 12 o'clock. Figs 19(a) and (b) show three-dimensional surface plots of the magnitude of the Fourier coefficients $P_{m n}(t)$ for the pressure at time $t=0$ and $t=1.5$, respectively, for the baseline simulation parameters. The higher Fourier coefficients have magnitudes that decay essentially to zero, indicating convergence. Although not shown here, an investigation into an appropriate grid resolution found that having greater that 161 grid points in each spatial variable produces contour plots that are for all intents and purposes identical to those using a grid that is 161 by 161 .
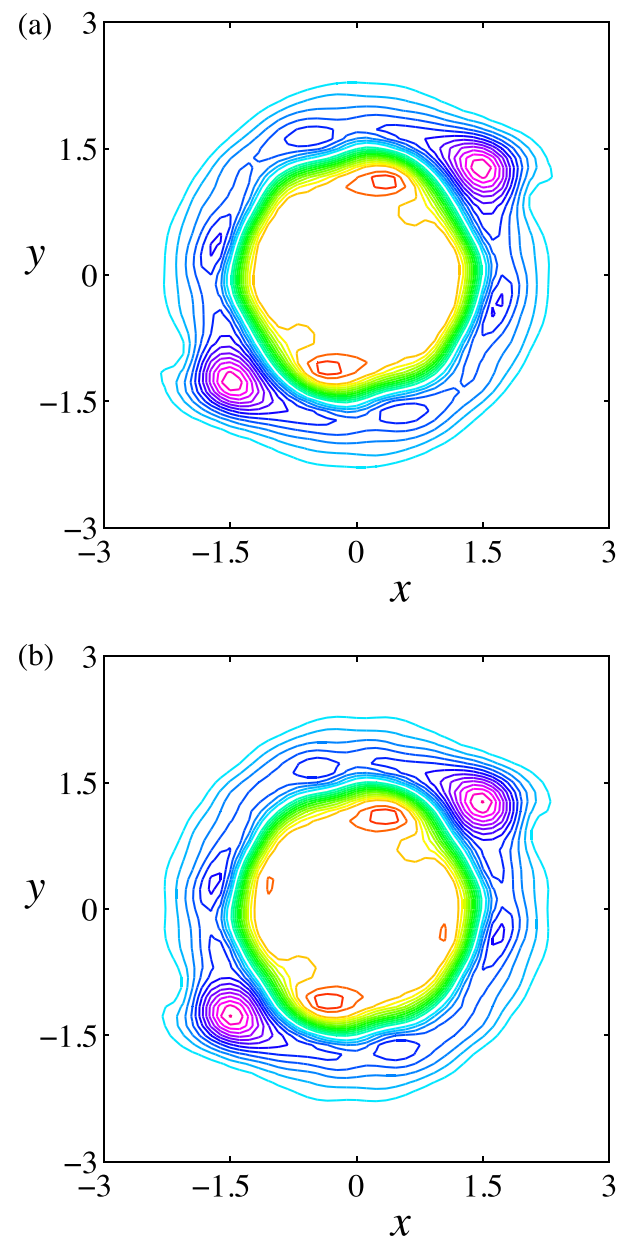

Figure 16. Pressure contour plots with two perturbing anticyclones located initially at $M=1.4$ of radius $\sigma=0.4$ and pressure differential $\mu=0.1$ at time $t=1.8$ using (a) Dirichlet boundary conditions and (b) Robin boundary conditions.

The contour diagrams above have been generated by considering the impact of two symmetrically located anticyclones perturbing an eastward circumpolar jet. In reality, there is a polar cyclone centred at the pole (Baines et al. 2009; Antuñano et al. 2015) and a visible anticyclone located in the interior of the hexagonal feature (Antuñano et al. 2015; Morales-Juberias et al. 2015). Although not shown here, a simulation of the polar cyclone alone does not produce any special feature and the associated pressure structure remains essentially circular. Incorporating an internal polar spot to the stand alone polar cyclone produces a distortion in the contour structure but never forms what could be interpreted as a hexagon. Including the polar cyclone in our simulations where there are two perturbing anticyclones seems to have marginal influence over the formation of the hexagon as indicated in Fig. 20, which illustrates the pressure contours of the baseline parameters at time $t=2.0$ with a polar cyclone component added to the background flow. There are small changes in the hexagonal feature compared to the baseline configuration shown previously in Fig. 10(c). Although slight, these differences may have been caused by increased shearing induced by the polar cyclone and thus produce closed pressure contour regions of similar length to the hexagonal edges, which are not evident in the contour plot in Fig. 10(c). An explanation for this similarity is that there is still a substantial ring within the hexagonal structure that is approximately stagnant and thus limits the effect of 
(a)

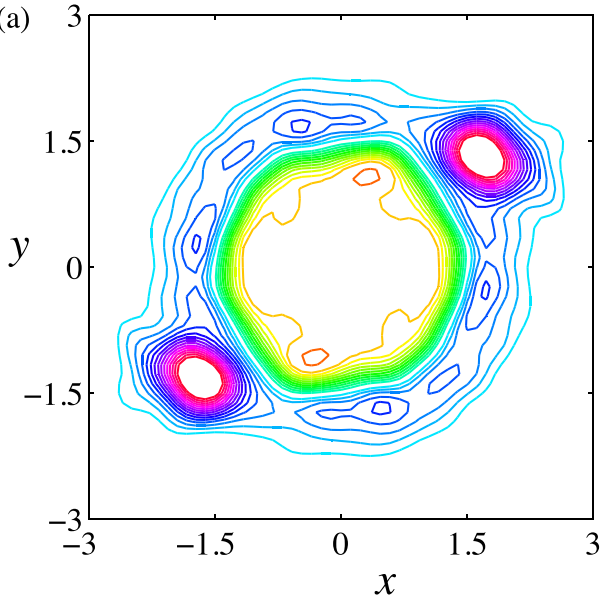

(b)

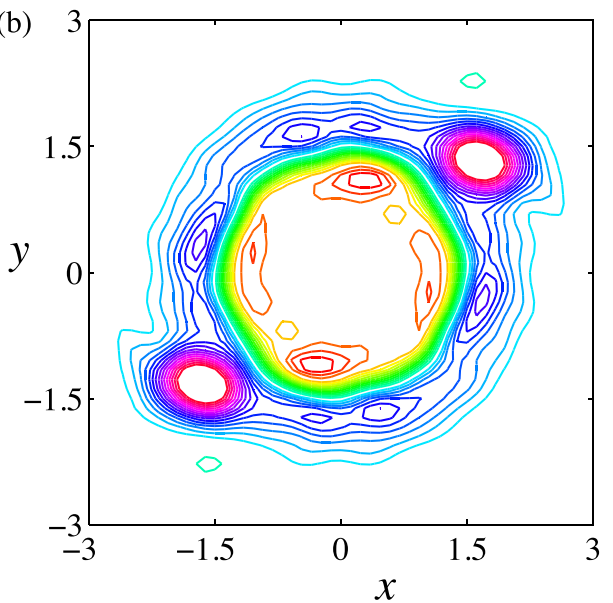

Figure 17. Pressure contour plots using baseline parameters with eastward jet having velocity (a) 75 per cent at time $t=2.2$ and (b) 125 per cent at time $t=1.8$.

the polar cyclone's influence on the hexagon formation. However, there are still flow-on effects from the polar cyclone components and although slight, these differences may have been caused by increased internal shearing induced by the polar cyclone.

\section{CONCLUSIONS AND DISCUSSION}

This paper has considered a possible trigger mechanism for the formation of the famed North Polar Hexagon on Saturn, in the form of perturbing anticyclones. The hexagonal feature is a long-lived atmospheric structure that continues to this day. It was theorized by Allison et al. (1990) that the hexagon structure was the result of a Rossby wave being perturbed by at least one anticyclone to the south. Although at this point in time there is no evidence of a perturbing anticyclone, the claim by Allison et al. (1990), in particular the perturbation aspect of the theory, has not been substantiated in the literature and thus is a focal point of this article. A high-latitude $\delta$-plane approximation is used with tangent point located at the north pole, $\phi=90^{\circ} \mathrm{N}$. In the $\delta$-plane approximation, the Coriolis acceleration varies quadratically with latitude.

The Hexagon is centrally located about $76^{\circ} \mathrm{N}$ latitude within an eastward circumpolar jet with an approximate maximum zonal velocity of $100 \mathrm{~m} \mathrm{~s}^{-1}$. There is a smaller magnitude, $20 \mathrm{~m} \mathrm{~s}^{-1}$, westward jet to the south at the approximate latitude of $69^{\circ} \mathrm{N}$ which seems to be ignored when modelling and simulating the formation
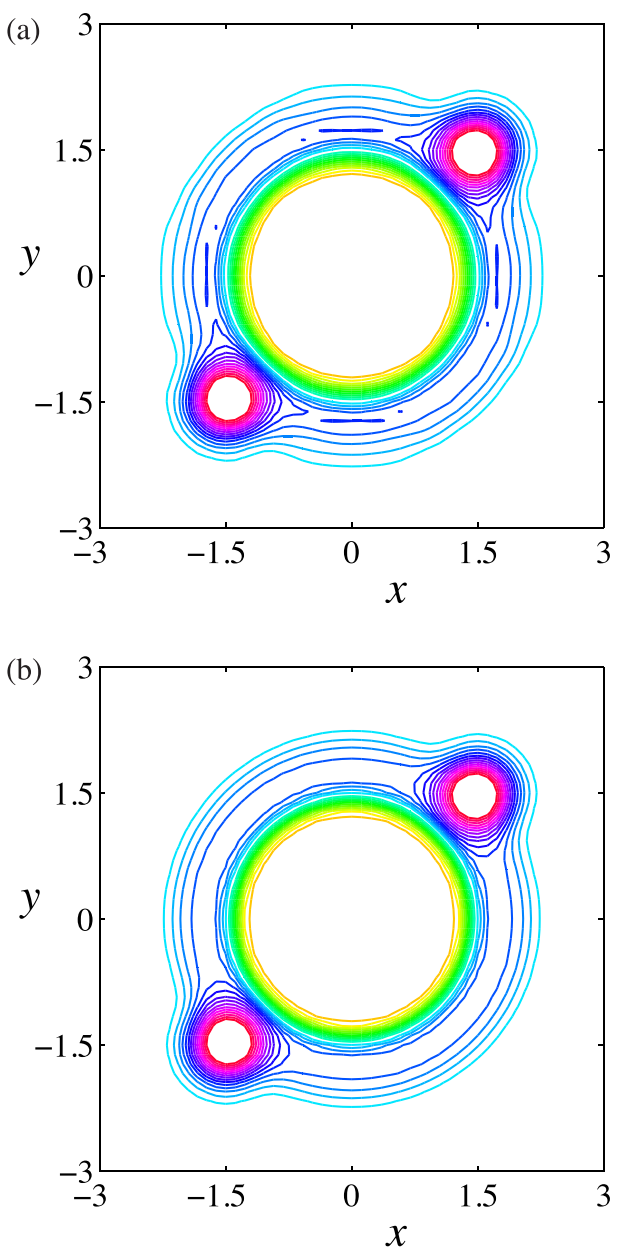

Figure 18. Initial pressure contour plots with two perturbing anticyclones located originally at $M=1.5$ of radius $\sigma=0.4$ with pressure differential $\mu=0.2$ for (a) spectral pressure and (b) 'true' representation.

of the Hexagon in the current literature. These circumpolar jets are simulated with a Gaussian profile function offset at the appropriate dimensionless distance from the pole. The perturbing anticyclones are modelled using an exponential function (19) with variable initial eye location $M$, radius $\sigma$ and pressure differential $\mu$. It was found that the most regular hexagonal structure formed at time $t=2.0$ using the parameters $M=1.5, \sigma=0.4$ and $\mu=0.2$. These were then used as the baseline values to determine the effect on hexagon formation of varying one of the parameters.

The pin-wheeling effect caused by the opposing rotations of the eastward jet and anticyclones being in close proximity is the major contributing factor in the formation of a hexagonal edge in the simulations. A result of moving the anticyclones closer to the jet is that the pin-wheeling may become non-tangential and a pronounced curve in the pressure contour structure can arise and cause the non-formation of a hexagonal vertex, and although a hexagonal structure may eventuate, it will never be regular. Conversely, if the anticyclones are moved away such that there is no pin-wheel effect occurring then an edge will never eventuate to form a hexagonal feature.

The pressure differential $\mu$ changes the internal velocity of the anticyclones and thus contributes to the pin-wheeling effect. Decreasing the pressure differential lessens the pin-wheeling effect between the polar jet and the anticyclones. To overcome this lack of 

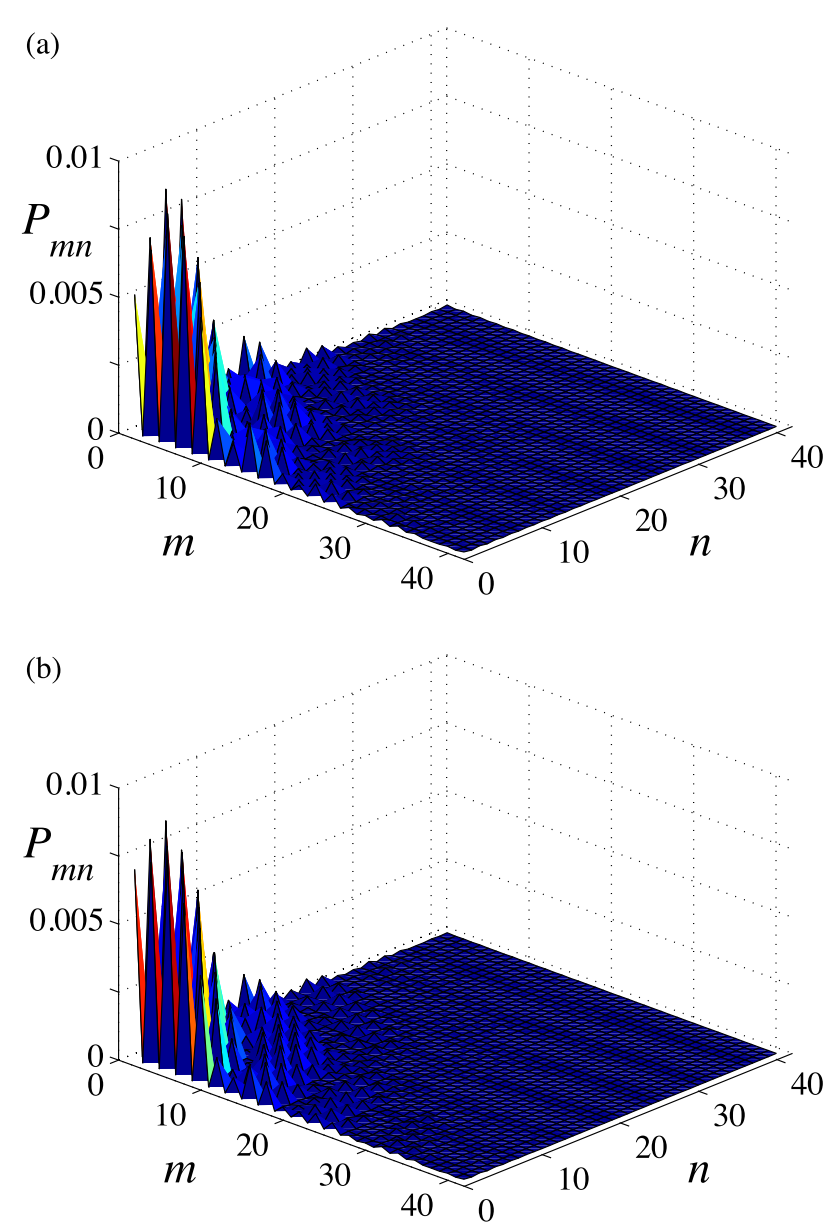

Figure 19. Absolute value of the Fourier coefficients $P_{m n}(t)$ for pressure with $M=1.5, \sigma=0.4$ and $\mu=0.2$ at time (a) $t=0$ and (b) $t=1.5$.

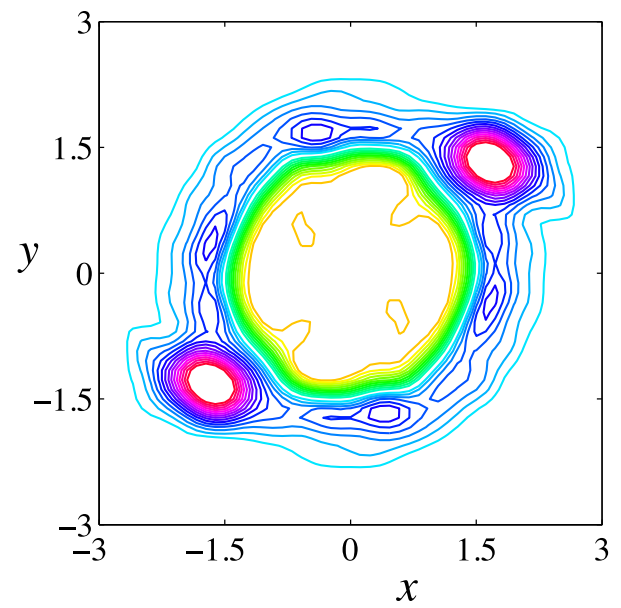

Figure 20. Pressure contour plot at time $t=2.0$ using baseline parameters with additional polar cyclone components added to the background flow.

influence, the location of the anticyclones must be moved closer to the circumpolar jet. Apart from the baseline parameters, it was also shown that a hexagonal structure is formed with decreased pressure differential $\mu=0.1$, closer location of $M=1.4$ and baseline radius of $\sigma=0.4$ at time $t=2.0$.
The radius of the anticyclone impacts on the internal velocity; if the pressure differential $\mu$ is identical, the smaller the radius of the anticyclone, the more intense the internal velocity. However, the anticyclone must be within a close enough proximity to influence the flow of the polar jet.

The initial unperturbed pressure profile of the circumpolar jet had pressure in the range $p=0.88$ to $p=1.04$. The intermediate pressure contour $p=0.96$ is used to define the hexagonal structure in the pressure contour maps. Surprisingly, the defining intermediate pressure value aligned itself extremely closely with the boundary between positive and negative local vorticity. Therefore, it could be concluded that the hexagonal feature may be linked through pressure and local vorticity.

It has been shown that a hexagonal feature can be formed through at least two anticyclones impinging on the southern boundary of the eastward circumpolar jet, with varying parameters, even with considerable smaller magnitude velocities than observed in nature. This is not a solution to the famed North Polar Hexagon on Saturn, but a demonstration of a trigger mechanism in the form of perturbing anticyclones that could help form a hexagonal structure. Our simulations produce hexagons that are transient, lasting approximately $t=1.5$ ( 2 days) in duration. We are unable to replicate the stability that has been observed in reality and thus the longevity of the structure has to be attributed to other factors not considered in this paper. This merits further research.

\section{ACKNOWLEDGEMENTS}

The work of the first author is supported by an Australian Government Research Training Program Scholarship. The authors are grateful for detailed and constructive comments from an anonymous reviewer.

\section{REFERENCES}

Allison M., Godfrey D. A., Beebe R. F., 1990, Science, 247, 1061

Antuñano A., del Rio-Gaztelurrutia T., Sanchez-Lavega A., Hueso R., 2015, J. Geophys. Res. Planets, 120, 155

Baines K. H. et al., 2009, Planet. Space Sci., 57, 1671

Barbosa-Aguiar A. C., Read P. L., Wordsworth R. D., Salter T., Yamazaki Y. H., 2010, Icarus, 206, 755

Caldwell J., Hua X., Turgeon B., Westphal J. A., Barnet C. D., 1993, Science, 260,326

Cosgrove J. M., Forbes L. K., 2017, J. Eng. Math., 104, 41

Godfrey D. A., 1988, Icarus, 76, 335

Godfrey D. A., 1990, Science, 247, 1206

Harlander U., 2005, Tellus, 57A, 43

Harlander U., Schonfeldt H.-J., Metz W., 2000, J. Geophys. Res., 105, 17063 Jansson T. R. N., Haspang M. P., Jensen K. H., Hersen P., Bohr T., 2006, Phys. Rev. Lett., 96, 174502

Kay J. M., Nedderman R. M., 1985, Fluid Mechanics and Transfer Processes. Cambridge Univ. Press, Cambridge

LeBlond P. H., 1964, Tellus, 16, 503

Lipps F. B., 1964, Tellus, 16, 535

Marcus P. S., Lee C., 1998, Phys. Fluids, 10, 1474

Morales-Juberias R., Sayanagi K. M., Dowling T. E., Ingersoll A. P., 2011, Icarus, 211,1284

Morales-Juberias R., Sayanagi K. M., Simon A. A., Fletcher L. N., Cosentino R. G., 2015, ApJ, 806, L18

Nof D., 1990, Geophys. Astrophys. Fluid Dyn., 52, 71

Sanchez-Lavega A., Lecacheux J., Colas F., Laques P., 1993, Science, 260, 329

Sanchez-Lavega A. et al., 2014, Geophys. Res. Lett., 41, 1425 
Sayanagi K. M., Ewald S. P., Ingersoll A. P., 2009, AAS/Division for Planetary Sciences Meeting Abstracts, 41, 10.08

Smith B. A. et al., 1977, Space Sci. Rev., 21, 103

Sommeria J., Meyers S. D., Swinney H. L., 1989, Nature, 337, 58

Vallis G. K., 2006, Atmospheric and Oceanic Fluid Dynamics. Cambridge Univ. Press, Cambridge

Vatistas G. H., 1990, J. Fluid Mech., 217, 241

Vatistas G. H., Wang J., Lin S., 1994, Acta Mech., 103, 89

von Winckel G., 2004, lgwt.m, at: MATLAB file exchange website. Available at: http://www.mathworks.com/matlabcentral/fileexchange/ loadFile.do?objectId=4540\&object Type $=$ file
Williams D. R., 2015, Saturn fact sheet, at: NASA website. Available at: http://nssdc.gsfc.nasa.gov/planetary/factsheet/saturnfact.html Yang H., 1987, J. Atmos. Sci., 44, 2267

This paper has been typeset from a $\mathrm{T}_{\mathrm{E}} \mathrm{X} / \mathrm{LAT} \mathrm{T}$ file prepared by the author. 